\title{
RR Lyrae Variables in the Crater II Dwarf Galaxy
}

\author{
Seok-Joo Joo ${ }^{1}$, Jaemann Kyeong ${ }^{1}$, Soung-Chul Yang ${ }^{1}$, Sang-Il Han ${ }^{1}$, Eon-Chang Sung ${ }^{1}$, Dongwon Kim ${ }^{2}$, Hyunjin Jeong ${ }^{1}$, \\ Chang H. Ree ${ }_{1}^{1}$, Soo-Chang Rey ${ }^{3}$, Helmut Jerjen ${ }^{4}$ (D), Hak-Sub Kim ${ }^{1}$, Sang-Mok Cha ${ }^{1,5}$, and Yongseok Lee ${ }^{1,5}$ \\ ${ }^{1}$ Korea Astronomy and Space Science Institute, Daejeon 34055, Republic of Korea; sjjoo@kasi.re.kr \\ ${ }^{2}$ Department of Astronomy, University of California, Berkeley, CA 94720-3411, USA \\ ${ }^{3}$ Department of Astronomy and Space Science, Chungnam National University, 99 Daehak-ro, Daejeon 34134, Republic of Korea \\ ${ }^{4}$ Research School of Astronomy and Astrophysics, Australian National University, Canberra, ACT 2611, Australia \\ ${ }^{5}$ School of Space Research, Kyung Hee University, Yongin, Kyeonggi 17104, Republic of Korea \\ Received 2018 March 29; revised 2018 May 4; accepted 2018 May 11; published 2018 June 27
}

\begin{abstract}
We report the detection of RR Lyrae variable stars in Crater II, a recently discovered large and diffuse satellite dwarf galaxy of the Milky Way (MW). Based on $B, V$ time-series photometry obtained with the Korea Microlensing Telescope Network $1.6 \mathrm{~m}$ telescope at Cerro Tololo Inter-American Observatory, we identified 83 $a b$-type and $13 c$-type pulsators by fitting template light curves. The detected RR Lyrae stars are centrally concentrated, which ensures that most of them are members of Crater II. In terms of the distribution of RRab stars in the period-amplitude diagram, Crater II is clearly different from ultra-faint dwarf (UFD) galaxies, but very similar to the two classical MW dwarf spheroidal (dSph) galaxies Draco and Carina with Oosterhoff-intermediate (Oo-int) properties. Combined with the mean period of $a b$-type variables $\left(\left\langle P_{a b}\right\rangle=0.631 \pm 0.004\right.$ days $)$ and the $c$-type fraction $(\sim 0.14)$ in Crater II, this suggests an Oo-int classification for Crater II and implies that its nature is more like a dSph rather than a UFD. We also estimated the mean metallicity, reddening, and distance of Crater II, from the photometric and pulsation properties of the RR Lyrae stars. The stellar population model we have constructed indicates that Crater II is dominated by an old population, but is relatively younger than the oldest globular clusters in the MW. With a lack of high-amplitude short-period RRab stars, Crater II, like most of the other less massive dSphs, is probably not a surviving counterpart of the major building blocks of the MW halo.
\end{abstract}

Key words: galaxies: dwarf - galaxies: individual (Crater II) - Local Group - stars: variables: RR Lyrae

\section{Introduction}

Over the past dozen years, the number of known satellite galaxies around the Milky Way (MW) has dramatically increased from 12 to $\sim 50$ (e.g., Willman et al. 2005a, 2005b; Belokurov et al. 2006, 2007, 2008, 2009, 2010; Grillmair 2006, 2009; Sakamoto \& Hasegawa 2006; Zucker et al. 2006a, 2006b; Irwin et al. 2007; Walsh et al. 2007; Bechtol et al. 2015; Drlica-Wagner et al. 2015, 2016; Kim et al. 2015a, 2015b; Kim \& Jerjen 2015; Koposov et al. 2015; Laevens et al. 2015a, 2015b; Martin et al. 2015; Torrealba et al. 2016a, 2016b; see also McConnachie 2012, updated 2015; Belokurov 2013 for reviews), thanks to large optical surveys such as the Sloan Digital Sky Survey (SDSS; York et al. 2000), the Dark Energy Survey (DES; DES Collaboration 2016), the Pan-STARRS1 Surveys (Chambers et al. 2016), and the VLT Survey Telescope (VST) ATLAS survey (Shanks et al. 2015). Most of these new MW dwarf companions are very faint in terms of both total luminosity $\left(M_{V} \gtrsim-8\right)$ and surface brightness $\left(\mu_{V} \gtrsim 28 \mathrm{mag} \operatorname{arcsec}^{-2}\right)$, leading to the term ultra-faint dwarf (UFD) galaxies. Spectroscopic and/or photometric follow-up studies (e.g., Muñoz et al. 2006, 2010; Martin et al. 2007; Simon \& Geha 2007; Kirby et al. 2008; Frebel et al. 2010; Norris et al. 2010; Simon et al. 2011; Brown et al. 2012, 2014; Okamoto et al. 2012; Sand et al. 2012; Koch \& Rich 2014; Kim et al. 2016a; Conn et al. 2018) in turn revealed that these least luminous galaxies are also most dark matter dominated (mass-to-light ratio, $M_{\odot} / L_{V \odot \odot} \gtrsim 100$ ), least chemically evolved $([\mathrm{Fe} / \mathrm{H}] \lesssim-2.5)$, and probably old $(t \gtrsim 10 \mathrm{Gyr})$. It is now naturally expected from the steep slope of the luminosity function of MW dwarf companions (Koposov et al. 2008) that the universe is dominated by these faintest galaxies in number.
The UFDs are believed to play an important role in resolving the well-known "missing satellite" problem, the discrepancy between observations and predictions from the current $\Lambda \mathrm{CDM}$ hierarchical merging paradigm, for the number and spatial distribution of satellite galaxies (Kauffmann et al. 1993; Klypin et al. 1999; Moore et al. 1999; see also Bullock \& Johnston 2005; Simon \& Geha 2007; Koposov et al. 2009; Bahl \& Baumgardt 2014). Hence, not only is a more complete census of MW dwarf satellites over the entire sky needed, but detailed studies of their stellar populations are also crucial to better understand their true nature, and thus galaxy formation and evolution via accretion and merger processes (e.g., Willman et al. 2004; Jerjen 2010; Conn et al. 2018).

The Crater II dwarf galaxy is one of the most recently discovered MW satellites in the southern hemisphere, first reported by Torrealba et al. (2016a) using the VST ATLAS survey data. From the total luminosity of $M_{V} \simeq-8.2$, Crater II can be classified as either one of the brightest UFDs or one of the faintest classical dwarf spheroidal (dSph) galaxies. Interestingly, given the total luminosity, this galaxy is very large (half-light radius, $\left.r_{h} \approx 1066 \mathrm{pc}\right)$ and diffuse $\left(\mu_{V} \approx 30.6 \mathrm{mag} \operatorname{arcsec}^{-2}\right)$. It is currently the fourth largest MW satellite-only the Large Magellanic Cloud (LMC), the Small Magellanic Cloud (SMC), and the Sagittarius (Sgr) dwarf galaxy are larger-and one of the lowest surface brightness galaxies known (Torrealba et al. 2016a, see their Figure 6). Based on the color-magnitude diagram (CMD) and isochrone fitting, Torrealba et al. (2016a) estimated Crater II to have an old age $(\sim 10 \mathrm{Gyr})$ and low metallicity $([\mathrm{Fe} / \mathrm{H}] \simeq-1.7)$. More recently, from MMT/Hectochelle spectroscopy, Caldwell et al. (2017) identified $\sim 62$ red giant branch (RGB) stars in this galaxy, and obtained a mean metallicity 


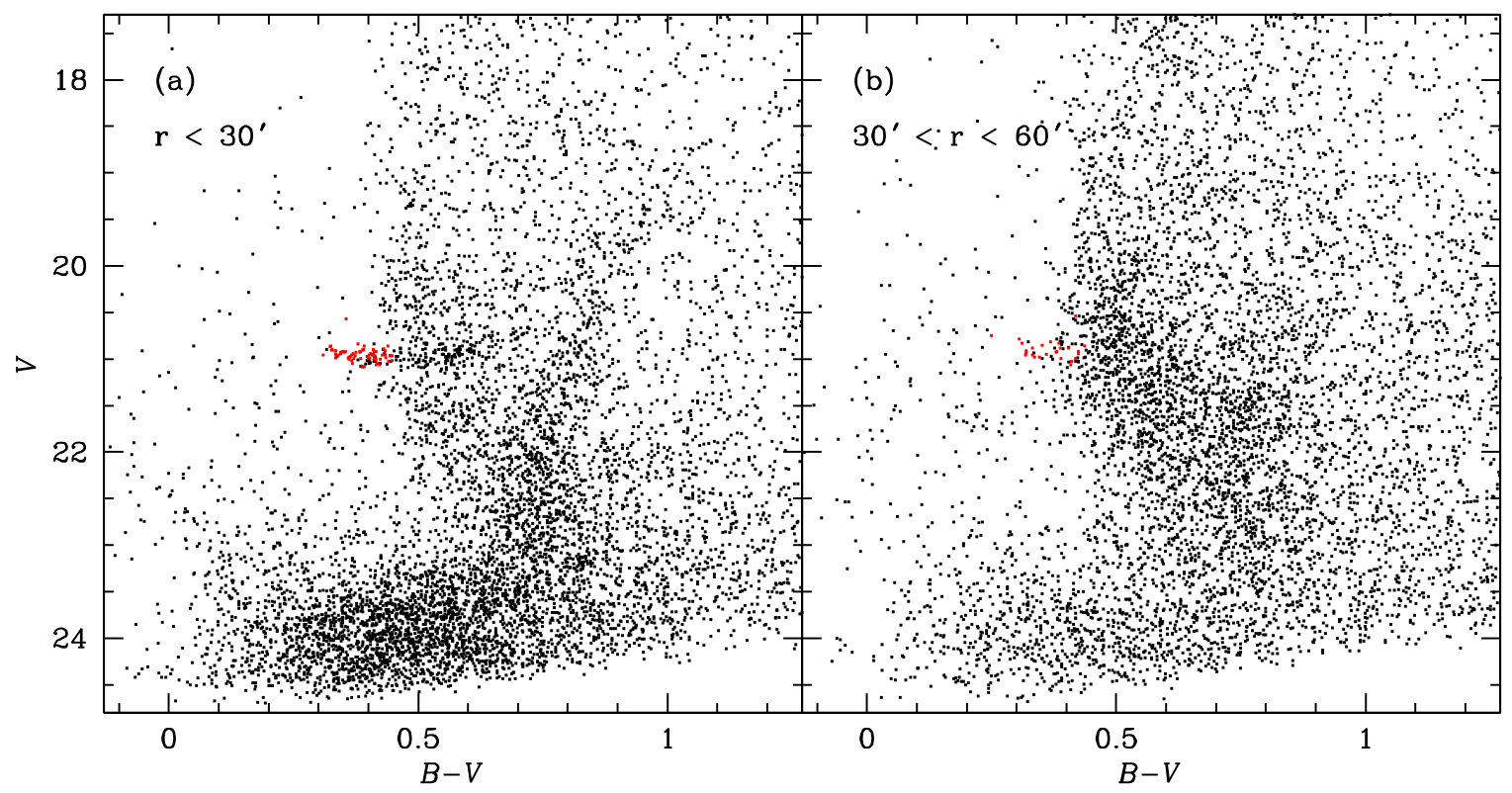

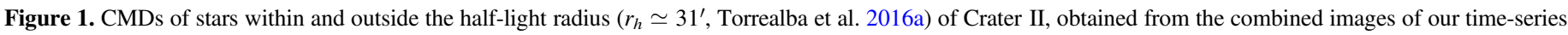

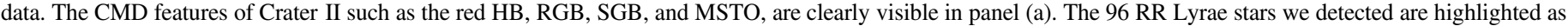
red points. See also Figure 4(b) for their spatial distribution.

of $\langle[\mathrm{Fe} / \mathrm{H}]\rangle \simeq-1.98$ with a dispersion of $\sigma_{[\mathrm{Fe} / \mathrm{H}]} \approx 0.22$. They also showed that it has an extremely low line-of-sight velocity dispersion $\left(\sigma_{v, \text { los }} \approx 2.7 \mathrm{~km} \mathrm{~s}^{-1}\right.$, see also McGaugh 2016) and a dynamical mass of $\sim 4.4 \times 10^{6} M_{\odot}$, suggesting a mass-to-light ratio of $\sim 53 M_{\odot} / L_{V, \odot}$ within the half-light radius. Located relatively distant from the Sun $(\gtrsim 100 \mathrm{kpc})$ and widely distributed on the sky ( $r_{h} \simeq 31^{\prime}$, Torrealba et al. 2016a), there is still a lack of extensive studies on the stellar population of Crater II.

As radially pulsating low-mass horizontal branch (HB) stars in the phase of core helium burning, RR Lyrae variables are good tracers of old ( $\gtrsim 10 \mathrm{Gyr}$ ) and metal-poor populations (i.e., population II). Their photometric and pulsation properties including mean magnitude, period, amplitude, and light curve, are commonly used to derive metallicity, interstellar reddening, and distance of the system. They can also provide information on the stellar structure, formation, and evolution of their host dwarf galaxies, particularly when compared with those stars in the Galactic halo or globular clusters (GCs) (e.g., Smith 2004; Catelan 2009; Clementini 2010; Pietrukowicz et al. 2015). Since 2006, about 13 UFDs (with $M_{V}>-7$ ) have been reported to contain RR Lyrae variables (Vivas et al. 2016, see their Table 4 for a recent compilation), including Boötes I (holding the current record with 15 RR Lyrae stars) and Segue I, one of the faintest UFDs known (Simon et al. 2011). It is interesting to note that all dwarf galaxies searched for variable stars so far have at least one RR Lyrae star (Vivas et al. 2016; see also Boettcher et al. 2013; Garofalo et al. 2013; Sesar et al. 2014; Medina et al. 2017) regardless of the total luminosity.

In this paper, we investigate the RR Lyrae population in the Crater II dwarf galaxy based on time-series observations using the Korea Microlensing Telescope Network (KMTNet) $1.6 \mathrm{~m}$ telescope located at the Cerro Tololo Inter-American Observatory (CTIO). This study is part of our ongoing southern hemisphere survey for RR Lyrae stars in UFDs using the KMTNet-CTIO. Section 2 presents the time-series observations and data reduction process. Section 3 describes our detection of the RR Lyrae stars by applying the template light curve fitting routine, RRFIT, developed by Yang \& Sarajedini (2012), and the characterization of the stars including their light curves, spatial distribution, and metallicity estimates. We discuss the results and draw our conclusions in Section 4.

\section{Observations and Data Reduction}

Time-series $B, V$ observations of the Crater II dwarf galaxy were carried out using the KMTNet-CTIO $1.6 \mathrm{~m}$ telescope during 2016 February 1-4, 6-10, and 2017 January 29-30 (UT). The telescope is equipped with the mosaic CCD camera of $18 \mathrm{k} \times 18 \mathrm{k}$ pixels, providing a wide field of view of $2^{\circ} \times 2^{\circ}$ and a pixel scale of $0{ }^{\prime \prime} 40$ (Kim et al. 2016b). Our observations covered a total area of $\sim 3^{\circ} \times 3^{\circ}$ with five largely overlapping fields, which is more than twice the half-light radius of the galaxy $\left(r_{h} \simeq 31^{\prime}\right.$, Torrealba et al. 2016a). With an exposure time of $120 \mathrm{~s}$ per image, we obtained in total 143 and 145 frames in the $B$ - and $V$-bands, respectively, which correspond to a total exposure time of $\sim 4.8 \mathrm{hr}$ in each passband.

All images were preprocessed by the KMTNet pipeline, which includes basic corrections such as cross-talk, bias subtraction, and flat fielding (Kim et al. 2016b). We performed point spread function (PSF) photometry using DAOPHOT II/ALLSTAR and ALLFRAME (Stetson 1987, 1994), similar to the previous works on Galactic GCs (Han et al. 2009b, 2015). Photometric calibration was achieved using the AAVSO Photometric All-Sky Survey (APASS) database (http://www. aavso.org/apass), and astrometric calibration was performed with SCAMP (Bertin 2006) applying the third US Naval Observatory (USNO) CCD Astrograph Catalog, UCAC3 (Zacharias et al. 2010).

The panels in Figure 1 show the CMDs of stars within and outside the half-light radius $\left(r_{h} \simeq 31^{\prime}\right)$ of Crater II, produced by performing PSF photometry on the combined images of all our time-series data with SWarp (Bertin et al. 2002). The CMDs were cleaned of poorly measured stars and non-stellar objects using the photometric errors and the ALLFRAME fitting parameters, $\mathrm{CHI}$ and SHARP, as functions of $B$ and $V$ 
magnitudes (see Kim et al. 2013; Lim et al. 2016). In panel (a), for stars within the half-light radius, we can clearly see the CMD features of Crater II, such as the RGB, red HB, sub-giant branch (SGB), and even main-sequence turn-off (MSTO). Panel (b), for stars outside the half-light radius, however, shows only weak features of Crater II superimposed on the background contamination, indicating that most member stars are within the half-light radius. Figure 1 generally confirms the CMD properties of Crater II presented by Torrealba et al. (2016a, see their Figure 1), but our observations are deep enough to reach the MSTO near $V \simeq 24$, which is important for age dating of stellar populations (see Section 4). The red dots represent RR Lyrae stars identified in this study, where their colors and magnitudes are mean values from our light curve analysis (see Section 3).

\section{RR Lyrae Variable Stars}

\subsection{Detection and Characterization}

To identify and characterize RR Lyrae stars in the field of Crater II, we applied the template light curve fitting routine, RRFIT, developed by Yang \& Sarajedini (2012) on the basis of template fitting methods by Layden (1998) and Mancone \& Sarajedini (2008). Following the technique of Yang et al. (2010, 2014) and Yang \& Sarajedini (2012), we first selected stars at the HB luminosity level, i.e., $20.2<V<21.7$, and examined their variability using the reduced chi-square, $\chi_{\nu}^{2}$, defined as

$$
\chi_{\nu}^{2}=\frac{1}{N_{B}+N_{V}} \times\left[\sum_{i=1}^{N_{B}} \frac{\left(B_{i}-\bar{B}\right)^{2}}{\sigma_{B i}^{2}}+\sum_{i=1}^{N_{V}} \frac{\left(V_{i}-\bar{V}\right)^{2}}{\sigma_{V i}^{2}}\right],
$$

where, for each star in the $B$ - and $V$-bands, $N_{B}$ and $N_{V}$ are the numbers of the observed frames, $B_{i}$ and $V_{i}$ are the apparent magnitudes in the $i_{\text {th }}$ images with uncertainties $\sigma_{B i}$ and $\sigma_{V i}$, and $\bar{B}$ and $\bar{V}$ are the mean magnitudes for the $N_{B}$ and $N_{V}$ frames. In the calculation of the $\chi_{\nu}^{2}$ value, data points further away than $3 \sigma$ from the mean magnitude were excluded. Variable stars are expected to have larger $\chi_{\nu}^{2}$ values than typical non-variable stars that have values around unity $\left(\chi_{\nu}^{2} \approx 1\right)$ in an ideal situation. We considered the stars with $\chi_{\nu}^{2}>3.0$ as potential variable candidates, to be about $30 \%$ of those selected earlier to be at the HB level.

We have then analyzed the time-series data of these variable candidates using RRFIT, where we rejected data points with magnitude error greater than 0.1 . Based on template light curves of RR Lyrae stars, RRFIT determines the best-fit light curve and parameter values such as period, amplitude, mean magnitude, and epoch of maximum light. As in the previous works by Yang et al. (2010, 2014) and Yang \& Sarajedini (2012), we used a library of 25 light curve templates, which are the six $a b$-type and two $c$-type templates from Layden \& Sarajedini (2000) and the $17 a b$-type templates from Kovács \& Kupi (2007). By visually inspecting the output light curves from RRFIT, we finally identified 96 RR Lyrae stars, including 83 fundamental mode ( $a b$-type) and 13 first overtone ( $c$-type) pulsators. ${ }^{6}$ Figure 2 presents the light curves and the best-fitting templates for all the detected RR Lyrae stars. Table 1 lists their pulsation properties, including coordinate, type, period, epoch

\footnotetext{
6 The faintest variable star detected in the field, V97, is not counted here as it is probably not an RR Lyrae star (see Section 3.3).
}

of maximum light, intensity-weighted mean magnitude $(\langle B\rangle$ and $\langle V\rangle)$, the number of observations $\left(N_{B}\right.$ and $\left.N_{V}\right)$, and amplitude $\left(A_{B}\right.$ and $\left.A_{V}\right)$. The mean magnitudes are obtained by averaging the intensity of the best-fit templates over 0.02 phase interval. The quantities $N_{B}$ and $N_{V}$ are the actual numbers of data points used in RRFIT, and some stars, for example those lying in the gaps between the CCDs, have fewer data points compared to stars detected in all observations.

\subsection{Synthetic Light Curve Simulation}

time-series analysis may suffer from aliasing (i.e., spurious periods), which is mainly caused by the limited number of observations, poor phase coverage, and/or photometric uncertainty. Hence, we need to scrutinize at what level the aliases affected the pulsation periods before using them to derive metallicity, reddening, and distance of Crater II. For this purpose, we performed synthetic light curve simulations and statistical tests following the prescription of Yang et al. (2014). Based on the light curve templates of Layden \& Sarajedini (2000) for RR Lyrae stars, we first generated $3000 a b$-type and $2000 c$-type artificial light curves by applying our observational constraints (such as number of epochs, cadence, observing baseline, and photometric errors), which were extracted from a few of the good RR Lyrae candidates. The (input) periods and amplitudes were randomly assigned to each artificial RR Lyrae star within the appropriate ranges of $a b$ - and $c$-type variables, respectively. We then ran RRFIT on these synthetic time-series data in the same way as for the observed ones, so that we can directly compare the assigned (i.e., input) and calculated (i.e., output) parameters.

The top and bottom panels in Figure 3 present the difference between input and output periods as a function of the input periods, for RRab and RRc stars, respectively. Our simulations show that $c$-type variables are more affected by aliasing than $a b$-type stars in the sense that the output periods are generally longer than the input periods. While the input periods of $\sim 91 \%$ of the synthetic RRab stars were recovered within \pm 0.05 day, only those of $\sim 27 \%$ of the artificial RRc stars were recovered within the same period range. Consequently, we have less confidence in the pulsation parameters calculated for the RRc stars in this study, and decided to use them only for the the mean magnitude and the $c$-type fraction (see Section 3.3).

We also estimated the systematic error in the mean period introduced by our period searching method based on statistical analysis. By employing the synthetic RR Lyrae (i.e., 3000 RRab and $2000 \mathrm{RRc}$ ) stars as a parent population, we randomly selected a subsample with the same number of stars as detected in our observation (i.e., 83 RRab and 13 RRc stars). Because each artificial RR Lyrae has a $\Delta P$ (input-output) value associated, we can calculate an average $\Delta P$ value for the subsample (i.e., $\left.\langle\Delta P\rangle_{i}\right)$. To increase the statistical significance, we repeated this random sampling 10,000 times and produced the $\langle\Delta P\rangle$ distribution for the 10,000 subsamples. We consider the $1 \sigma$ range of the $\langle\Delta P\rangle$ distribution from the best-fit Gaussian as a good estimate for the systematic error in the mean period of the RR Lyrae stars derived by our template light curve analysis. We find a systematic error, $\sigma_{\left\langle P_{a b}\right\rangle}= \pm 0.0018$ day for the mean period of the RRab stars. 

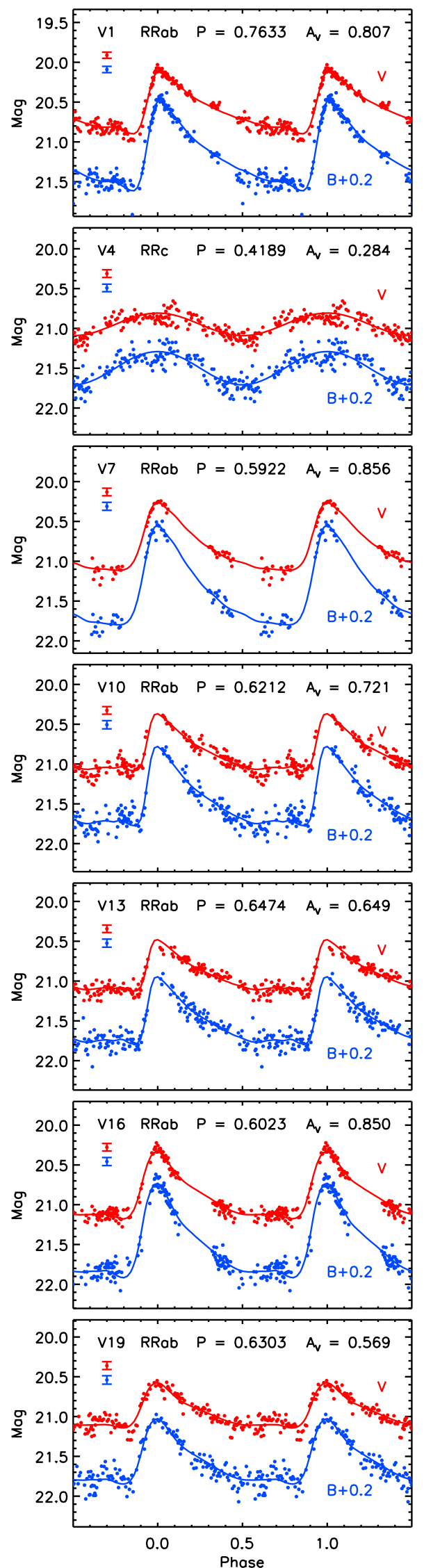
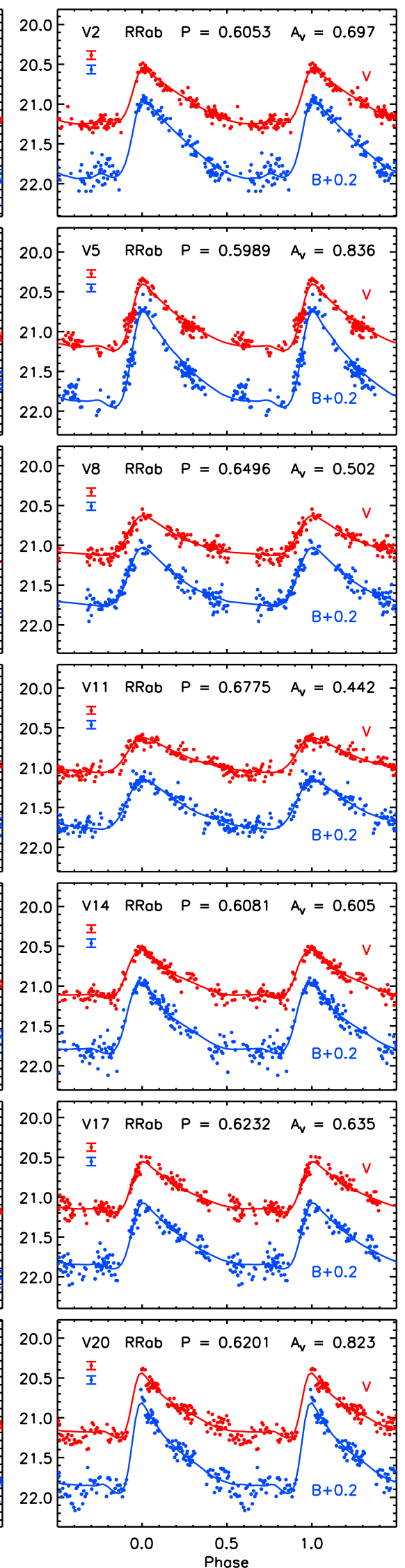
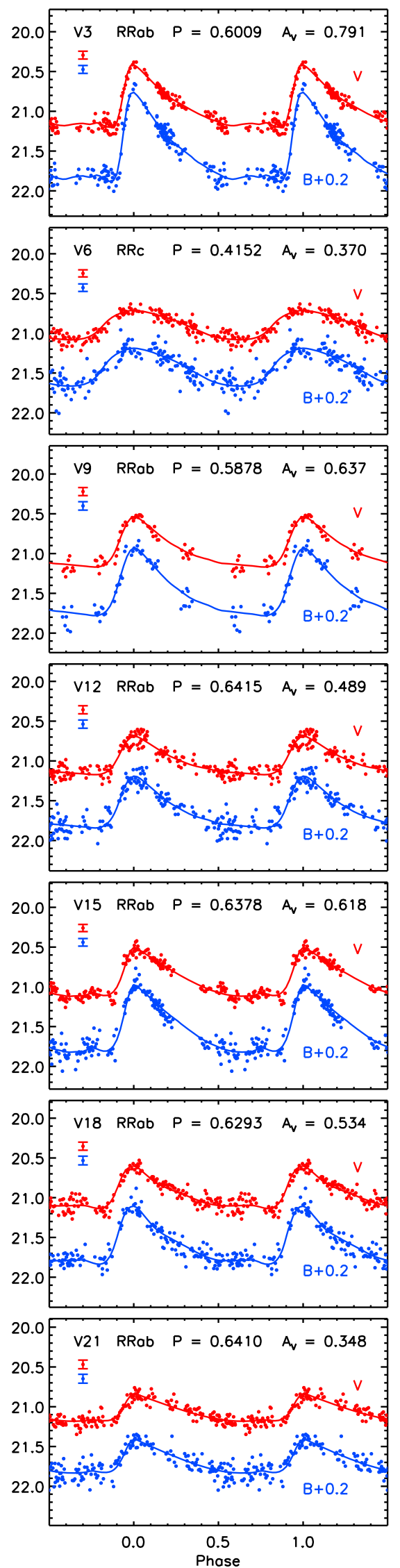

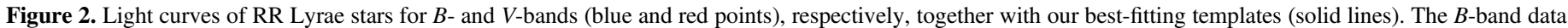

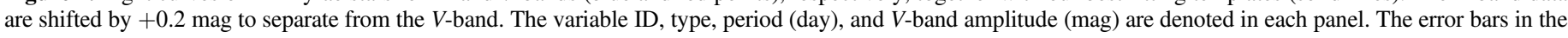
upper left corner represent the mean photometric errors for each band. 

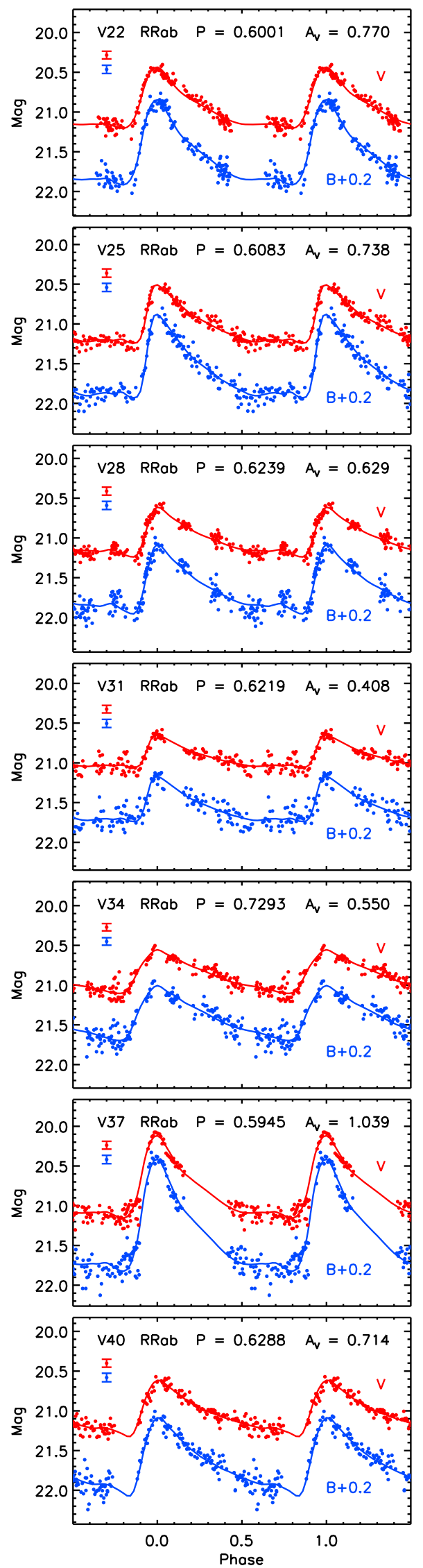
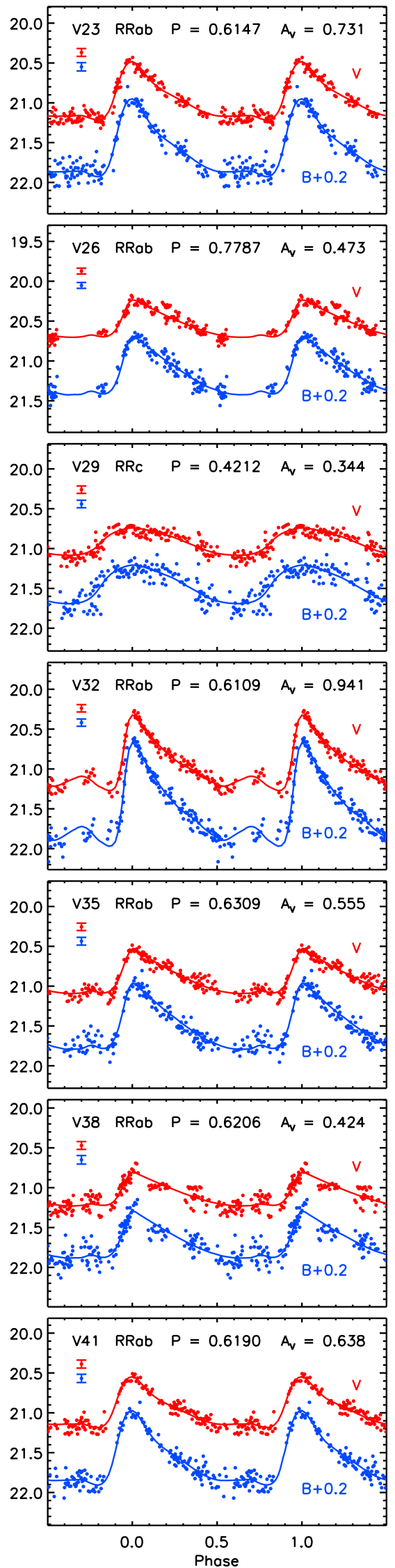

Figure 2. (Continued.)
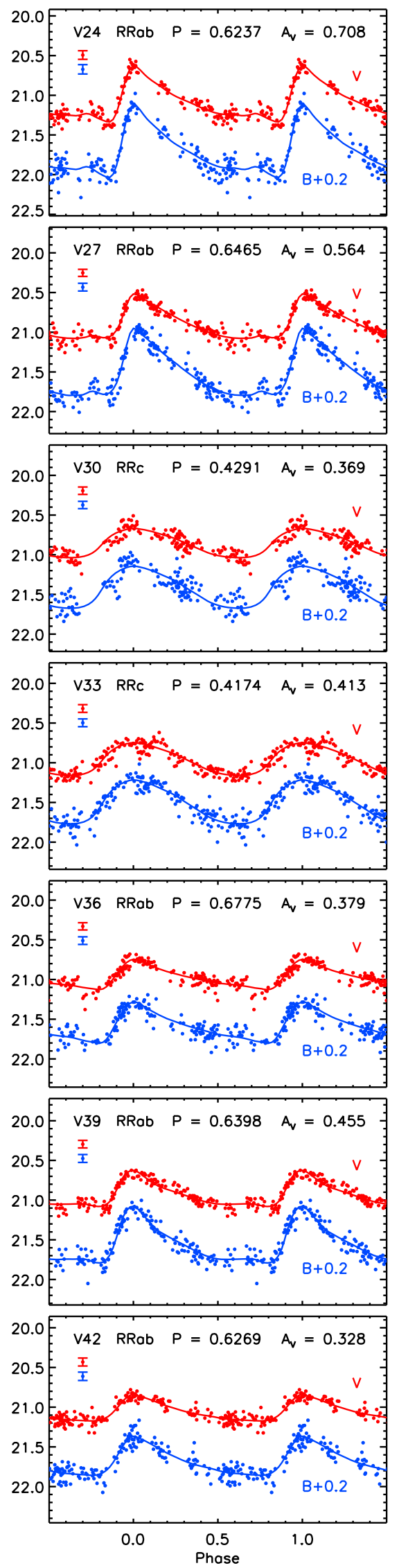

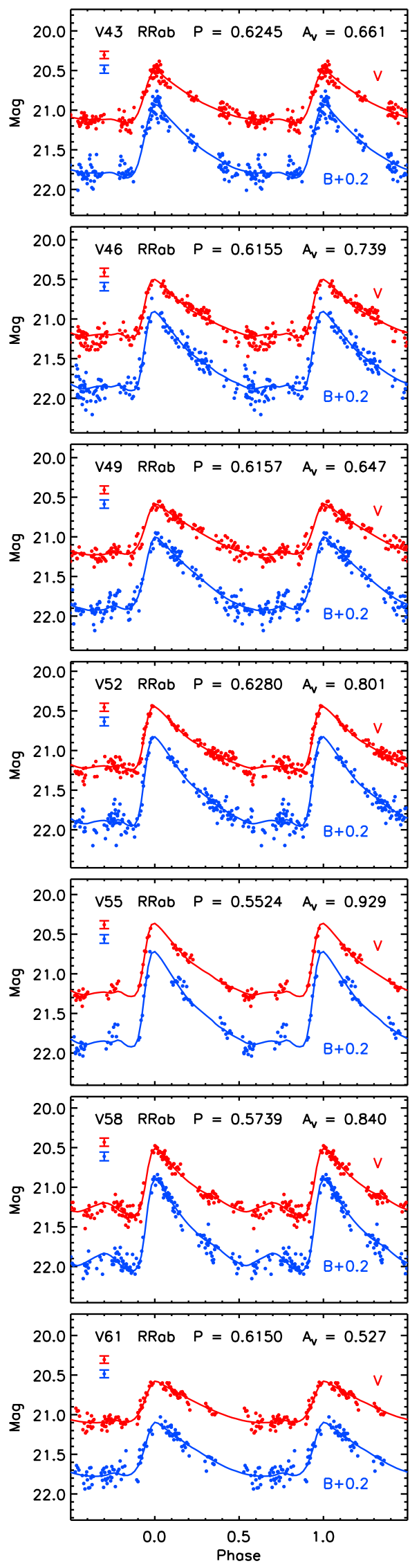


Figure 2. (Continued.)
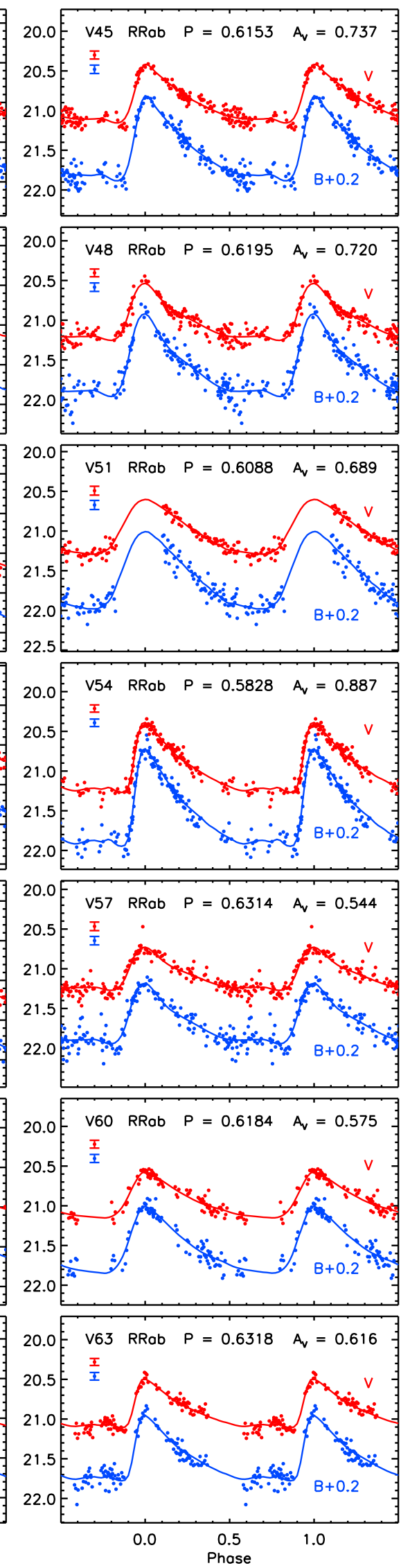

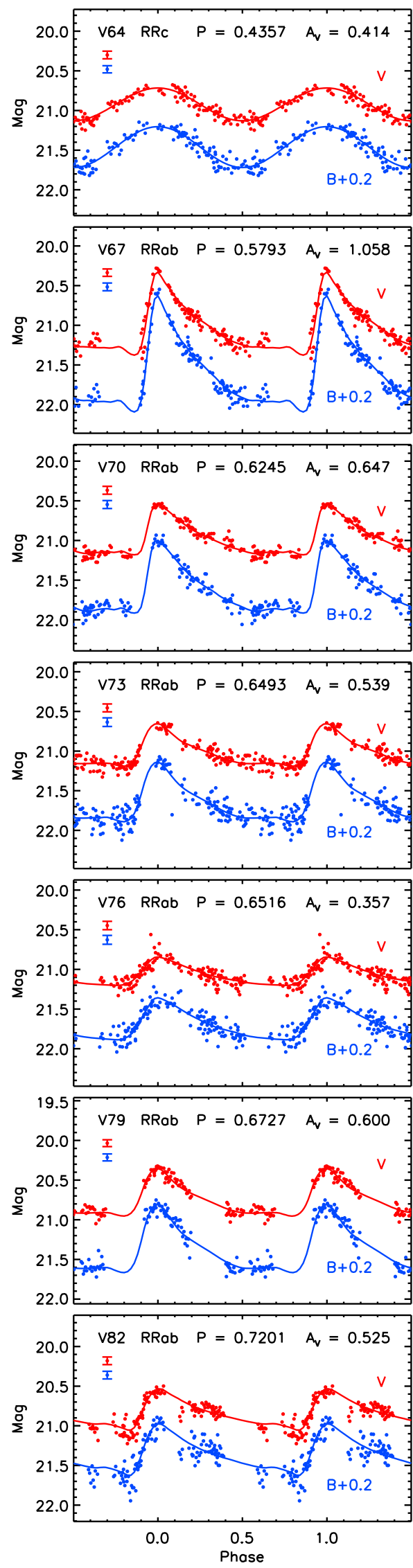
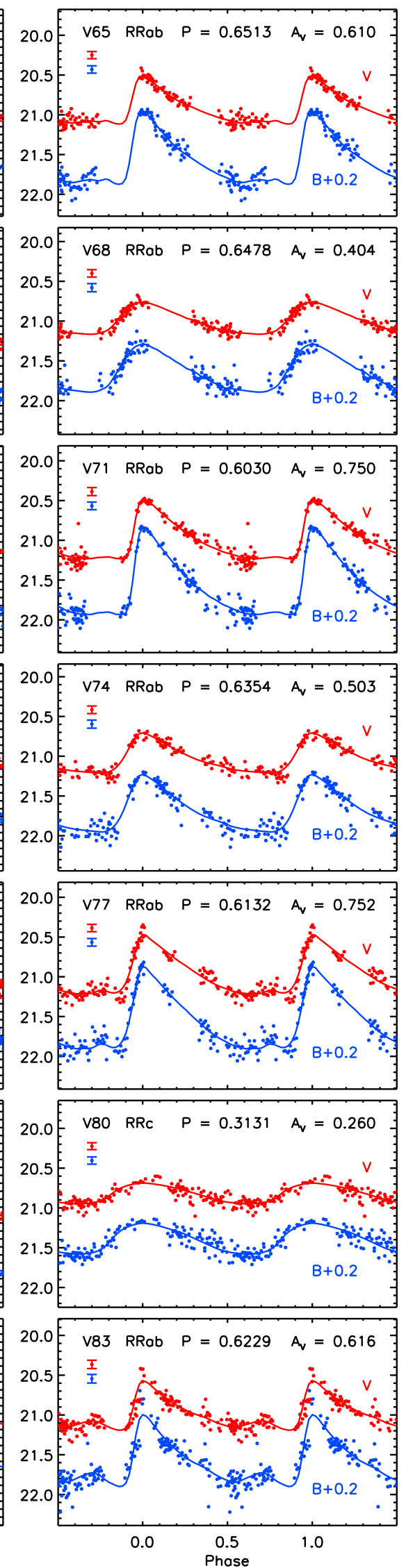

Figure 2. (Continued.)
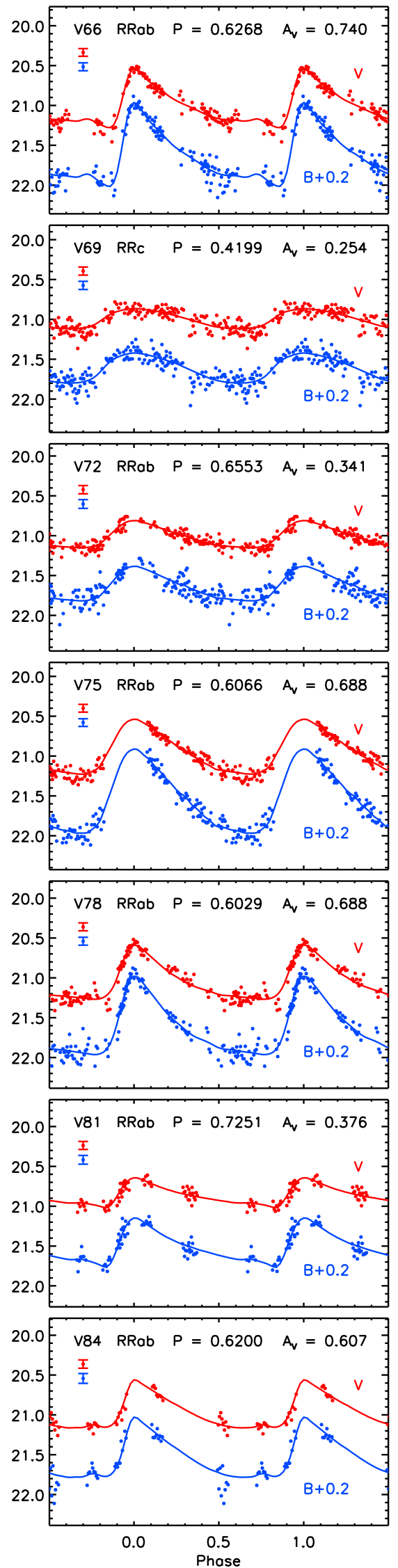

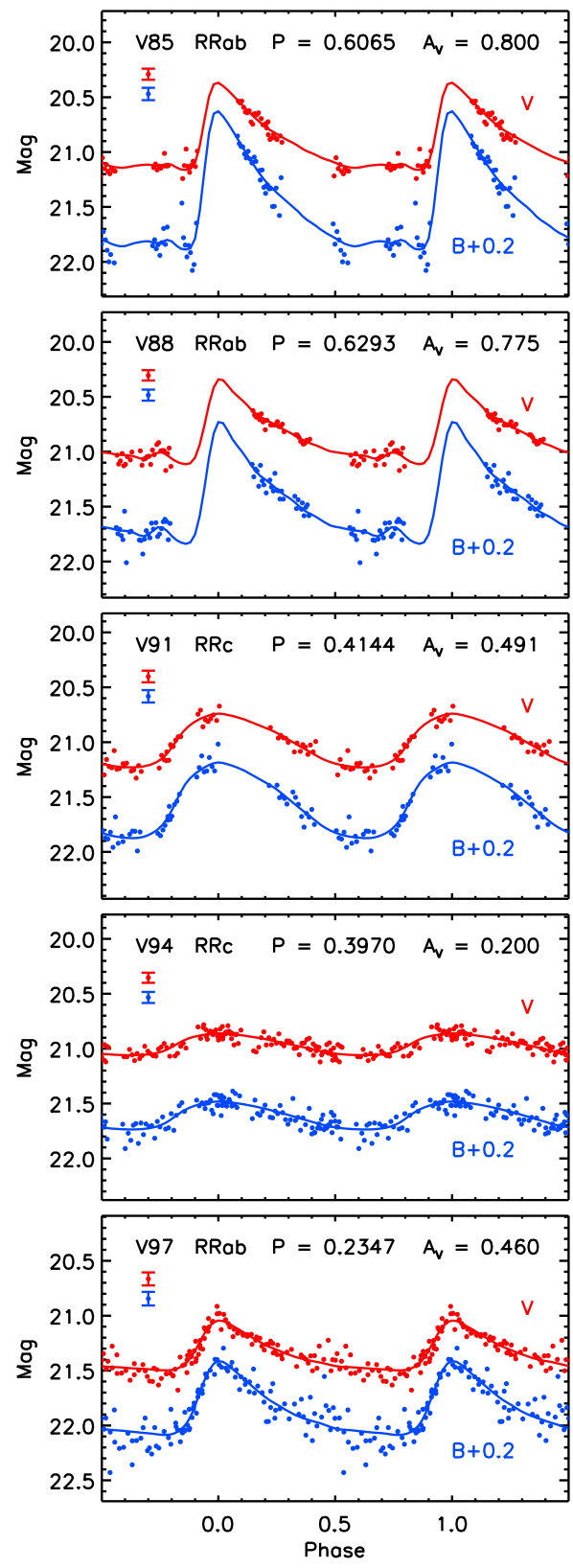
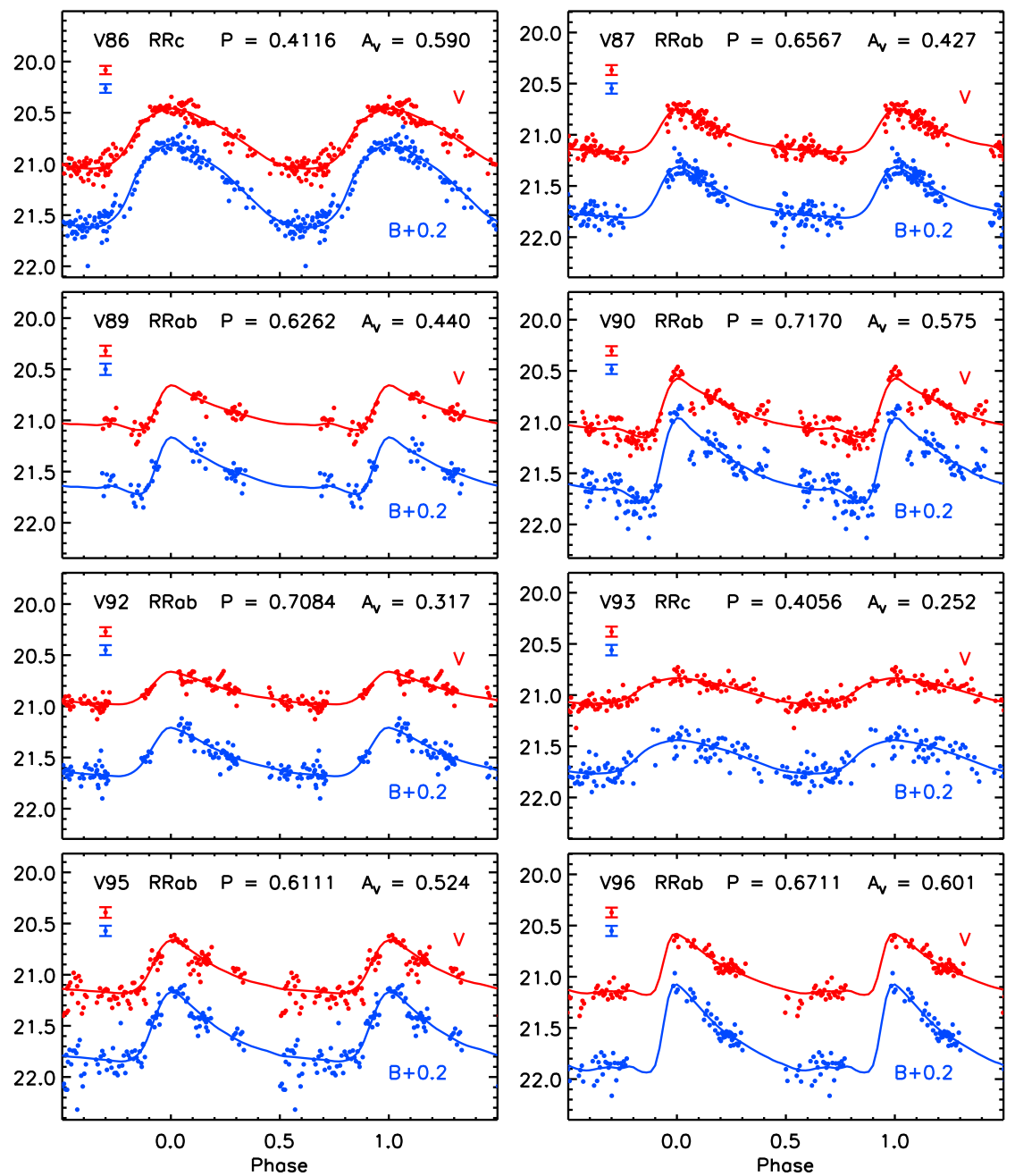

Figure 2. (Continued.)

\subsection{CMD and Spatial Distribution}

The panels in Figure 4 present the CMD, spatial distribution, and color-period diagram of the RR Lyrae stars we have detected in Crater II. The two gray vertical lines in the upper panels denote the empirical instability strip, roughly estimated by averaging the blue and red boundaries of nine Galactic and LMC clusters from Walker (1998, see Table 7 in that paper). The boundaries were reddened by assuming an interstellar extinction value of $E(B-V)=0.05$ mag (see Section 3.6). It is clear from panel (a) and Figure 1 that most RR Lyrae stars are within the appropriate color range of the instability strip at the level of the HB. Panel (a) also shows that the RR Lyrae stars gather on the red side of the instability strip in their color distribution. This further indicates that Crater II has a red HB morphology with virtually no blue HB stars, which leads the HB morphology index of Lee et al. (1994),
$(B-R) /(B+V+R)$, to be roughly $\lesssim-0.5$, where $B, V$, and $R$ are the numbers of blue HB, variable (RR Lyrae) and red HB stars, respectively. This red HB morphology naturally explains the relatively small number of $c$-type variables compared to that of $a b$-type stars, yielding the $c$-type fraction to all RR Lyrae variables, $N(c) / N(a b+c) \simeq 0.14$.

Panel (b) illustrates that the RR Lyrae stars are clustered around the galaxy's center estimated by Torrealba et al. (2016a). The two histograms on the top and right sides of the panel demonstrate that they are centrally well concentrated. About 70\% (67 out of 96) of RR Lyrae stars are within 30' (approximately the half-light radius), and about 97\% (93 out of 96) of them are within $60^{\prime}$ from the center. The CMD and spatial distribution of the RR Lyrae variables in panels (a) and (b) thus strongly suggest that most of them belong to Crater II. The large heliocentric distance of the galaxy $(\sim 112 \mathrm{kpc}$, see Section 3.6) further ensures this interpretation, because the 
Table 1

Pulsation Properties of RR Lyrae Stars in Crater II

\begin{tabular}{|c|c|c|c|c|c|c|c|c|c|c|c|c|}
\hline ID & $\begin{array}{l}\text { R.A. } \\
\text { (2000) }\end{array}$ & $\begin{array}{l}\text { Decl. } \\
(2000)\end{array}$ & Type & $\begin{array}{l}\text { Period } \\
\text { (days) }\end{array}$ & $\begin{array}{l}\text { Epoch (max) } \\
(-2457000)\end{array}$ & $\begin{array}{c}\langle B\rangle^{\mathrm{i}} \\
(\mathrm{mag})\end{array}$ & $\begin{array}{c}\langle V\rangle^{\mathrm{i}} \\
(\mathrm{mag})\end{array}$ & $N_{B}{ }^{\mathrm{ii}}$ & $N_{V}^{\mathrm{ii}}$ & $\begin{array}{c}A_{B}^{\mathrm{iii}} \\
(\mathrm{mag})\end{array}$ & $\begin{array}{l}A_{V}^{\mathrm{iii}} \\
(\mathrm{mag})\end{array}$ & Note \\
\hline $\mathrm{V} 1$ & $11: 48: 46.95$ & $-18: 37: 28.97$ & $a b$ & 0.7633 & 525.8221 & 20.921 & 20.565 & 141 & 144 & 1.155 & 0.807 & Field? ? $^{\text {iv }}$ \\
\hline V2 & 11:48:59.49 & $-18: 10: 09.97$ & $a b$ & 0.6053 & 762.3792 & 21.360 & 20.999 & 136 & 142 & 1.000 & 0.697 & $\cdots$ \\
\hline V3 & 11:48:38.76 & $-18: 31: 40.36$ & $a b$ & 0.6009 & 528.5124 & 21.269 & 20.925 & 138 & 144 & 1.128 & 0.791 & $\cdots$ \\
\hline V4 & 11:48:51.17 & $-18: 16: 34.26$ & $c$ & 0.4189 & 765.8670 & 21.289 & 20.945 & 142 & 145 & 0.418 & 0.284 & $\cdots$ \\
\hline V5 & $11: 48: 51.88$ & $-18: 31: 56.98$ & $a b$ & 0.5989 & 526.3172 & 21.277 & 20.926 & 141 & 144 & 1.205 & 0.836 & $\cdots$ \\
\hline V6 & 11:49:04.42 & $-18: 02: 02.16$ & $c$ & 0.4152 & 759.0594 & 21.224 & 20.897 & 138 & 141 & 0.476 & 0.370 & $\cdots$ \\
\hline V7 & $11: 48: 30.82$ & $-17: 41: 58.38$ & $a b$ & 0.5922 & 422.8492 & 21.088 & 20.782 & 54 & 54 & 1.251 & 0.856 & $\cdots$ \\
\hline V8 & $11: 48: 47.00$ & $-18: 31: 30.74$ & $a b$ & 0.6496 & 569.1721 & 21.288 & 20.944 & 139 & 144 & 0.746 & 0.502 & $\ldots$ \\
\hline V9 & 11:46:00.64 & $-18: 49: 38.18$ & $a b$ & 0.5878 & 421.0890 & 21.257 & 20.937 & 51 & 54 & 0.860 & 0.637 & $\cdots$ \\
\hline V10 & 11:47:51.72 & $-18: 11: 12.19$ & $a b$ & 0.6212 & 704.6499 & 21.215 & 20.836 & 140 & 144 & 1.000 & 0.721 & $\cdots$ \\
\hline V11 & $11: 48: 47.71$ & $-17: 58: 10.30$ & $a b$ & 0.6775 & 598.5794 & 21.319 & 20.888 & 141 & 144 & 0.632 & 0.442 & $\cdots$ \\
\hline V12 & $11: 48: 41.01$ & $-18: 31: 45.59$ & $a b$ & 0.6415 & 673.1422 & 21.401 & 20.998 & 139 & 144 & 0.658 & 0.489 & $\cdots$ \\
\hline V13 & 11:48:51.60 & $-18: 41: 34.13$ & $a b$ & 0.6474 & 530.1833 & 21.292 & 20.903 & 139 & 144 & 0.849 & 0.649 & $\cdots$ \\
\hline V14 & $11: 48: 39.63$ & $-18: 09: 24.26$ & $a b$ & 0.6081 & 437.9900 & 21.315 & 20.931 & 139 & 143 & 0.895 & 0.605 & $\cdots$ \\
\hline V15 & $11: 47: 57.03$ & $-18: 08: 39.69$ & $a b$ & 0.6378 & 488.0886 & 21.309 & 20.899 & 141 & 141 & 0.852 & 0.618 & $\cdots$ \\
\hline V16 & $11: 48: 33.63$ & $-18: 04: 30.77$ & $a b$ & 0.6023 & 431.0587 & 21.254 & 20.862 & 140 & 144 & 1.189 & 0.850 & $\cdots$ \\
\hline V17 & $11: 47: 34.18$ & $-18: 39: 23.29$ & $a b$ & 0.6232 & 648.7332 & 21.391 & 20.957 & 140 & 144 & 0.828 & 0.635 & $\cdots$ \\
\hline V18 & 11:47:33.17 & $-18: 06: 30.50$ & $a b$ & 0.6293 & 684.8898 & 21.369 & 20.945 & 136 & 143 & 0.733 & 0.534 & $\cdots$ \\
\hline V19 & 11:48:04.16 & $-18: 04: 44.49$ & $a b$ & 0.6303 & 476.6949 & 21.344 & 20.939 & 137 & 144 & 0.824 & 0.569 & $\cdots$ \\
\hline V20 & $11: 48: 35.40$ & $-18: 30: 54.83$ & $a b$ & 0.6201 & 523.9000 & 21.325 & 20.959 & 137 & 144 & 1.152 & 0.823 & $\cdots$ \\
\hline V21 & 11:49:05.76 & $-18: 32: 03.12$ & $a b$ & 0.6410 & 440.5893 & 21.487 & 21.064 & 140 & 143 & 0.425 & 0.348 & $\cdots$ \\
\hline V22 & 11:48:14.91 & $-18: 15: 09.20$ & $a b$ & 0.6001 & 525.2433 & 21.306 & 20.922 & 137 & 143 & 1.071 & 0.770 & $\cdots$ \\
\hline V23 & 11:48:10.64 & $-18: 17: 42.81$ & $a b$ & 0.6147 & 677.0265 & 21.357 & 20.947 & 139 & 142 & 0.980 & 0.731 & $\cdots$ \\
\hline V24 & $11: 48: 42.45$ & $-19: 10: 16.40$ & $a b$ & 0.6237 & 575.8158 & 21.470 & 21.061 & 120 & 126 & 0.917 & 0.708 & $\cdots$ \\
\hline V25 & 11:49:05.84 & $-18: 40: 47.01$ & $a b$ & 0.6083 & 637.7355 & 21.347 & 20.987 & 134 & 144 & 1.058 & 0.738 & $\cdots$ \\
\hline V26 & $11: 51: 45.15$ & $-18: 29: 39.52$ & $a b$ & 0.7787 & 493.0874 & 20.958 & 20.540 & 124 & 126 & 0.743 & 0.473 & Field $?^{\text {iv }}$ \\
\hline V27 & $11: 50: 26.35$ & $-17: 59: 03.83$ & $a b$ & 0.6465 & 737.8831 & 21.284 & 20.879 & 141 & 143 & 0.851 & 0.564 & $\cdots$ \\
\hline V28 & 11:50:15.89 & $-18: 09: 38.24$ & $a b$ & 0.6239 & 739.1900 & 21.409 & 20.998 & 139 & 144 & 0.879 & 0.629 & $\cdots$ \\
\hline V29 & 11:49:23.50 & $-18: 20: 08.62$ & $c$ & 0.4212 & 768.2114 & 21.248 & 20.914 & 141 & 144 & 0.485 & 0.344 & $\cdots$ \\
\hline V30 & $11: 52: 15.27$ & $-17: 59: 36.36$ & $c$ & 0.4291 & 687.0483 & 21.205 & 20.853 & 123 & 126 & 0.532 & 0.369 & $\cdots$ \\
\hline V31 & $11: 51: 45.12$ & $-18: 07: 32.99$ & $a b$ & 0.6219 & 592.6235 & 21.344 & 20.920 & 122 & 126 & 0.574 & 0.408 & $\cdots$ \\
\hline V32 & $11: 50: 28.96$ & $-18: 16: 44.18$ & $a b$ & 0.6109 & 697.0341 & 21.270 & 20.922 & 141 & 144 & 1.287 & 0.941 & $\cdots$ \\
\hline V33 & 11:49:45.66 & $-18: 32: 56.41$ & $c$ & 0.4174 & 467.0077 & 21.288 & 20.953 & 142 & 144 & 0.555 & 0.413 & $\cdots$ \\
\hline V34 & 11:50:44.94 & $-18: 35: 15.51$ & $a b$ & 0.7293 & 752.6376 & 21.183 & 20.858 & 141 & 144 & 0.692 & 0.550 & Evolved? \\
\hline V35 & 11:49:56.30 & $-18: 37: 43.16$ & $a b$ & 0.6309 & 422.9369 & 21.288 & 20.898 & 142 & 145 & 0.836 & 0.555 & $\cdots$ \\
\hline V36 & 11:51:01.26 & $-18: 32: 21.17$ & $a b$ & 0.6775 & 510.8891 & 21.377 & 20.964 & 141 & 143 & 0.517 & 0.379 & $\cdots$ \\
\hline V37 & 11:53:04.48 & $-17: 55: 31.25$ & $a b$ & 0.5945 & 707.3147 & 21.058 & 20.759 & 121 & 124 & 1.414 & 1.039 & Field? \\
\hline V38 & $11: 50: 41.80$ & $-18: 40: 20.12$ & $a b$ & 0.6206 & 648.1834 & 21.475 & 21.083 & 140 & 143 & 0.593 & 0.424 & $\cdots$ \\
\hline V39 & 11:49:28.96 & $-18: 01: 34.50$ & $a b$ & 0.6398 & 777.5090 & 21.330 & 20.919 & 142 & 144 & 0.715 & 0.455 & $\cdots$ \\
\hline V40 & $11: 49: 46.30$ & $-18: 41: 45.65$ & $a b$ & 0.6288 & 527.1416 & 21.425 & 21.011 & 137 & 143 & 0.995 & 0.714 & $\cdots$ \\
\hline V41 & 11:50:03.59 & $-18: 44: 29.89$ & $a b$ & 0.6190 & 503.8013 & 21.355 & 20.954 & 138 & 143 & 0.937 & 0.638 & $\cdots$ \\
\hline V42 & 11:49:60.00 & $-18: 05: 32.04$ & $a b$ & 0.6269 & 508.8869 & 21.466 & 21.047 & 141 & 144 & 0.489 & 0.328 & $\cdots$ \\
\hline V43 & $11: 50: 41.04$ & $-18: 16: 30.60$ & $a b$ & 0.6245 & 602.3398 & 21.304 & 20.921 & 140 & 144 & 0.925 & 0.661 & $\cdots$ \\
\hline V44 & 11:49:50.68 & $-18: 45: 00.70$ & $a b$ & 0.6555 & 652.6502 & 21.464 & 21.023 & 141 & 144 & 0.817 & 0.559 & $\cdots$ \\
\hline V45 & $11: 50: 36.21$ & $-17: 57: 29.45$ & $a b$ & 0.6153 & 500.0881 & 21.271 & 20.883 & 140 & 144 & 1.071 & 0.737 & $\ldots$ \\
\hline V46 & $11: 49: 36.82$ & $-18: 35: 26.76$ & $a b$ & 0.6155 & 733.8168 & 21.343 & 20.980 & 141 & 144 & 1.006 & 0.739 & $\cdots$ \\
\hline V47 & 11:50:28.10 & $-17: 52: 38.65$ & $a b$ & 0.6199 & 744.8254 & 21.380 & 20.991 & 97 & 99 & 0.629 & 0.413 & $\cdots$ \\
\hline V48 & $11: 50: 29.54$ & $-18: 43: 02.85$ & $a b$ & 0.6195 & 654.8936 & 21.362 & 20.991 & 135 & 144 & 1.045 & 0.720 & $\ldots$ \\
\hline V49 & 11:50:07.96 & $-18: 41: 46.42$ & $a b$ & 0.6157 & 696.5972 & 21.382 & 20.997 & 140 & 143 & 0.932 & 0.647 & $\cdots$ \\
\hline V50 & 11:49:46.73 & $-18: 32: 13.11$ & $a b$ & 0.5877 & 674.4818 & 21.228 & 20.902 & 140 & 145 & 1.440 & 1.055 & $\cdots$ \\
\hline V51 & $11: 50: 13.18$ & $-18: 59: 39.05$ & $a b$ & 0.6088 & 708.1373 & 21.327 & 20.981 & 94 & 99 & 0.974 & 0.689 & $\cdots$ \\
\hline V52 & 11:49:40.59 & $-18: 19: 04.64$ & $a b$ & 0.6280 & 467.7285 & 21.337 & 20.963 & 139 & 142 & 1.133 & 0.801 & $\cdots$ \\
\hline V53 & 11:50:25.11 & $-18: 44: 06.21$ & $a b$ & 0.5717 & 562.2589 & 21.295 & 20.955 & 138 & 144 & 1.264 & 0.963 & $\cdots$ \\
\hline V54 & 11:52:17.11 & $-18: 34: 52.91$ & $a b$ & 0.5828 & 558.2980 & 21.289 & 20.957 & 123 & 126 & 1.235 & 0.887 & $\cdots$ \\
\hline V55 & 11:51:48.10 & $-18: 37: 45.56$ & $a b$ & 0.5524 & 420.8955 & 21.275 & 20.957 & 61 & 62 & 1.210 & 0.929 & $\cdots$ \\
\hline V56 & $11: 49: 44.86$ & $-18: 35: 02.55$ & $a b$ & 0.5658 & 622.0854 & 21.266 & 20.956 & 139 & 144 & 1.329 & 1.023 & $\cdots$ \\
\hline V57 & $11: 49: 53.69$ & $-18: 16: 39.62$ & $a b$ & 0.6314 & 466.5745 & 21.465 & 21.077 & 139 & 145 & 0.765 & 0.544 & $\cdots$ \\
\hline V58 & 11:50:06.34 & $-18: 45: 52.32$ & $a b$ & 0.5739 & 519.9465 & 21.418 & 21.050 & 136 & 143 & 1.148 & 0.840 & $\cdots$ \\
\hline V59 & 11:49:14.69 & $-18: 46: 04.99$ & $c$ & 0.4440 & 716.7583 & 21.193 & 20.870 & 106 & 109 & 0.543 & 0.363 & $\cdots$ \\
\hline V60 & 11:47:09.71 & $-18: 22: 15.93$ & $a b$ & 0.6184 & 747.3337 & 21.304 & 20.923 & 86 & 90 & 0.838 & 0.575 & $\ldots$ \\
\hline V61 & $11: 49: 10.58$ & $-18: 01: 03.85$ & $a b$ & 0.6150 & 509.6780 & 21.332 & 20.916 & 105 & 109 & 0.685 & 0.527 & $\cdots$ \\
\hline V62 & $11: 50: 22.85$ & $-18: 24: 50.27$ & $a b$ & 0.6106 & 648.3371 & 21.303 & 20.928 & 106 & 109 & 0.805 & 0.593 & $\cdots$ \\
\hline V63 & $11: 50: 30.79$ & $-18: 21: 32.67$ & $a b$ & 0.6318 & 577.9878 & 21.287 & 20.898 & 104 & 108 & 0.829 & 0.616 & $\ldots$ \\
\hline
\end{tabular}


Table 1

(Continued)

\begin{tabular}{|c|c|c|c|c|c|c|c|c|c|c|c|c|}
\hline ID & $\begin{array}{l}\text { R.A. } \\
\text { (2000) }\end{array}$ & $\begin{array}{l}\text { Decl. } \\
(2000)\end{array}$ & Type & $\begin{array}{l}\text { Period } \\
\text { (days) }\end{array}$ & $\begin{array}{c}\text { Epoch (max) } \\
(-2457000)\end{array}$ & $\begin{array}{c}\langle B\rangle^{\mathrm{i}} \\
(\mathrm{mag})\end{array}$ & $\begin{array}{c}\langle V\rangle^{\mathrm{i}} \\
(\mathrm{mag})\end{array}$ & $N_{B}^{\mathrm{ii}}$ & $N_{V}^{\mathrm{ii}}$ & $\begin{array}{c}A_{B}^{\mathrm{iiii}} \\
(\mathrm{mag})\end{array}$ & $\begin{array}{c}A_{V}{ }^{\mathrm{iiii}} \\
(\mathrm{mag})\end{array}$ & Note \\
\hline V64 & 11:50:18.67 & $-18: 24: 41.02$ & $c$ & 0.4357 & 623.2801 & 21.246 & 20.913 & 105 & 107 & 0.516 & 0.414 & $\cdots$ \\
\hline V65 & $11: 47: 45.05$ & $-18: 23: 26.99$ & $a b$ & 0.6513 & 426.8200 & 21.343 & 20.909 & 104 & 109 & 0.930 & 0.610 & $\ldots$ \\
\hline V66 & 11:49:47.00 & $-18: 27: 20.97$ & $a b$ & 0.6268 & 691.0083 & 21.390 & 20.990 & 105 & 108 & 1.057 & 0.740 & $\cdots$ \\
\hline V67 & $11: 49: 08.33$ & $-18: 48: 13.60$ & $a b$ & 0.5793 & 508.5757 & 21.323 & 20.989 & 103 & 107 & 1.480 & 1.058 & $\cdots$ \\
\hline V68 & $11: 49: 12.51$ & $-18: 19: 36.59$ & $a b$ & 0.6478 & 584.6497 & 21.424 & 20.989 & 105 & 104 & 0.601 & 0.404 & $\cdots$ \\
\hline V69 & 11:50:42.38 & $-18: 45: 42.65$ & $c$ & 0.4199 & 748.9645 & 21.411 & 20.993 & 142 & 144 & 0.378 & 0.254 & Less certain \\
\hline V70 & 11:49:12.04 & $-18: 15: 50.36$ & $a b$ & 0.6245 & 734.3474 & 21.388 & 20.960 & 104 & 108 & 0.924 & 0.647 & $\cdots$ \\
\hline V71 & 11:50:15.91 & $-18: 25: 53.60$ & $a b$ & 0.6030 & 566.5885 & 21.336 & 20.972 & 104 & 108 & 1.093 & 0.750 & $\cdots$ \\
\hline V72 & 11:48:49.99 & $-18: 28: 27.59$ & $a b$ & 0.6553 & 590.3775 & 21.428 & 21.004 & 138 & 143 & 0.433 & 0.341 & $\cdots$ \\
\hline V73 & $11: 49: 30.22$ & $-18: 41: 32.33$ & $a b$ & 0.6493 & 425.8456 & 21.414 & 20.999 & 141 & 143 & 0.754 & 0.539 & $\ldots$ \\
\hline V74 & 11:50:03.94 & $-18: 26: 10.76$ & $a b$ & 0.6354 & 480.2623 & 21.460 & 21.013 & 105 & 108 & 0.720 & 0.503 & $\ldots$ \\
\hline V75 & 11:50:06.00 & $-18: 24: 33.33$ & $a b$ & 0.6066 & 455.4853 & 21.271 & 20.917 & 105 & 108 & 1.057 & 0.688 & $\cdots$ \\
\hline V76 & 11:49:04.08 & $-18: 09: 07.73$ & $a b$ & 0.6516 & 605.3905 & 21.479 & 21.064 & 141 & 145 & 0.527 & 0.357 & $\cdots$ \\
\hline V77 & 11:49:17.38 & $-18: 38: 55.86$ & $a b$ & 0.6132 & 609.9599 & 21.313 & 20.943 & 104 & 109 & 1.043 & 0.752 & $\cdots$ \\
\hline V78 & 11:49:16.91 & $-18: 33: 54.38$ & $a b$ & 0.6029 & 494.6237 & 21.382 & 21.015 & 105 & 109 & 0.996 & 0.688 & $\cdots$ \\
\hline V79 & $11: 54: 43.85$ & $-17: 54: 41.34$ & $a b$ & 0.6727 & 497.5064 & 21.146 & 20.737 & 87 & 90 & 0.861 & 0.600 & Field? \\
\hline V80 & 11:51:51.56 & $-18: 30: 52.50$ & $c$ & 0.3131 & 452.9847 & 21.186 & 20.818 & 123 & 126 & 0.388 & 0.260 & Less certain \\
\hline V81 & 11:47:08.25 & $-18: 52: 29.86$ & $a b$ & 0.7251 & 428.6111 & 21.293 & 20.856 & 61 & 62 & 0.619 & 0.376 & Evolved? \\
\hline V82 & $11: 47: 09.17$ & $-18: 32: 22.45$ & $a b$ & 0.7201 & 771.3640 & 21.140 & 20.829 & 121 & 126 & 0.655 & 0.525 & Evolved? \\
\hline V83 & $11: 48: 45.47$ & $-18: 09: 58.75$ & $a b$ & 0.6229 & 484.0740 & 21.369 & 20.972 & 139 & 143 & 0.896 & 0.616 & $\cdots$ \\
\hline V84 & $11: 46: 43.04$ & $-17: 48: 50.38$ & $a b$ & 0.6200 & 421.7759 & 21.308 & 20.948 & 36 & 36 & 0.761 & 0.607 & $\cdots$ \\
\hline V85 & $11: 46: 02.28$ & $-18: 32: 12.10$ & $a b$ & 0.6065 & 425.7883 & 21.216 & 20.884 & 52 & 54 & 1.270 & 0.800 & $\ldots$ \\
\hline V86 & 11:50:57.17 & $-18: 46: 03.17$ & $c$ & 0.4116 & 478.0126 & 20.994 & 20.745 & 142 & 145 & 0.814 & 0.590 & $\cdots$ \\
\hline V87 & 11:51:07.05 & $-18: 39: 26.04$ & $a b$ & 0.6567 & 451.0808 & 21.431 & 21.021 & 140 & 144 & 0.502 & 0.427 & $\cdots$ \\
\hline V88 & $11: 49: 45.80$ & $-17: 38: 45.38$ & $a b$ & 0.6293 & 425.1559 & 21.213 & 20.828 & 54 & 54 & 1.113 & 0.775 & $\cdots$ \\
\hline V89 & 11:50:49.88 & $-17: 41: 56.21$ & $a b$ & 0.6262 & 426.0486 & 21.309 & 20.929 & 54 & 54 & 0.560 & 0.440 & $\cdots$ \\
\hline V90 & 11:52:06.09 & $-18: 44: 09.16$ & $a b$ & 0.7170 & 498.2968 & 21.235 & 20.915 & 122 & 126 & 0.819 & 0.575 & Evolved? \\
\hline V91 & 11:51:28.16 & $-18: 44: 26.91$ & $c$ & 0.4144 & 425.4381 & 21.318 & 20.982 & 50 & 51 & 0.690 & 0.491 & $\cdots$ \\
\hline V92 & 11:48:58.04 & $-18: 22: 07.78$ & $a b$ & 0.7084 & 782.6699 & 21.297 & 20.858 & 104 & 109 & 0.473 & 0.317 & $\cdots$ \\
\hline V93 & $11: 48: 24.47$ & $-18: 23: 10.60$ & $c$ & 0.4056 & 524.7682 & 21.405 & 20.962 & 104 & 109 & 0.327 & 0.252 & Less certain \\
\hline V94 & 11:49:17.63 & $-18: 06: 02.86$ & $c$ & 0.3970 & 556.2699 & 21.410 & 20.962 & 105 & 109 & 0.255 & 0.200 & Less certain \\
\hline V95 & 11:50:58.40 & $-18: 27: 02.71$ & $a b$ & 0.6111 & 538.9536 & 21.395 & 20.993 & 105 & 106 & 0.687 & 0.524 & Blend? \\
\hline V96 & 11:52:54.07 & $-19: 28: 31.18$ & $a b$ & 0.6711 & 759.5477 & 21.432 & 20.974 & 78 & 81 & 0.866 & 0.601 & Field? \\
\hline V97 & $11: 49: 16.15$ & $-18: 18: 35.54$ & $a b ?$ & 0.2347 & 439.4544 & 21.636 & 21.334 & 104 & 105 & 0.681 & 0.460 & Field DC? \\
\hline
\end{tabular}

Notes.

${ }^{\mathrm{i}}$ Intensity-weighted mean magnitude.

ii Number of observations used in our light curve analysis.

iii Pulsation amplitude.

iv Field? Highly evolved? AC?

Galactic halo RR Lyrae stars are thought to be rare at distances greater than $\sim 80 \mathrm{kpc}$ (Watkins et al. 2009; Zinn et al. 2014; Vivas et al. 2016). The three stars (cyan open circles) located more than $60^{\prime}\left(\gtrsim 2 r_{h}\right.$ ) from the center, on the other hand, are also among the brightest (V37, V79) or the reddest (V96) variables in the CMD. These three outliers might be field stars, and are denoted as "Field?" in Table 1.

In panel (c), we plot the fundamental periods $\left(P_{f}\right)$ of the RR Lyrae stars as a function of $B-V$ color, where the periods of RRc stars were fundamentalized assuming the period ratio between the $c$-type and $a b$-type stars, $P_{c} / P_{a b}=0.745$ (Nemec 1985; Clement et al. 2001). While most RR Lyrae stars follow a tight correlation between the fundamental period and color, some stars (green symbols) have considerably longer or shorter periods at a given color compared to the other stars. The two $a b$-type stars with the longest periods, V1 and V26 (green filled circles), are also the most luminous ( $\sim 0.4$ mag brighter than the bulk of the variables) as shown in panel (a). Given their periods and luminosity, these two stars appear to be either field RR Lyrae stars or highly evolved RR Lyrae stars from the zero-age HB (see, e.g., Lee et al. 1990).
Otherwise, they might be faint anomalous Cepheids (ACs) (Pritzl et al. 2002; Soszyński et al. 2008). The four $a b$-type stars with long periods (green open circles) can be seen as evolved RR Lyrae stars, since they are also relatively bright in the CMD. The four $c$-type stars with short periods (green open triangles), however, are less certain. They are too red to be $c$-type stars (V69, V93, and V94) or have somewhat short periods (V80). These four $c$-type stars might be field stars or influenced by other factors such as photometric errors or aliasing. The 10 outliers in panel (c) are represented in Table 1 as either "Field? Highly evolved? AC?", "Evolved?", or "Less certain", according to their properties.

For all the 96 RR Lyrae stars, we obtain the mean apparent magnitude, $\left\langle V_{\mathrm{RR}}\right\rangle=20.95 \pm 0.01 \mathrm{mag}$, by fitting a Gaussian profile at the magnitude distribution, where the uncertainty is the standard error of the mean. Note that, even if the 13 outliers (the three and 10 outliers in panels (b) and (c), respectively) are excluded, $\left\langle V_{\mathrm{RR}}\right\rangle$ does not change.

The faintest variable, V97 (black circle), in panel (a) is also distinct from the other stars. It is not only separated from the 

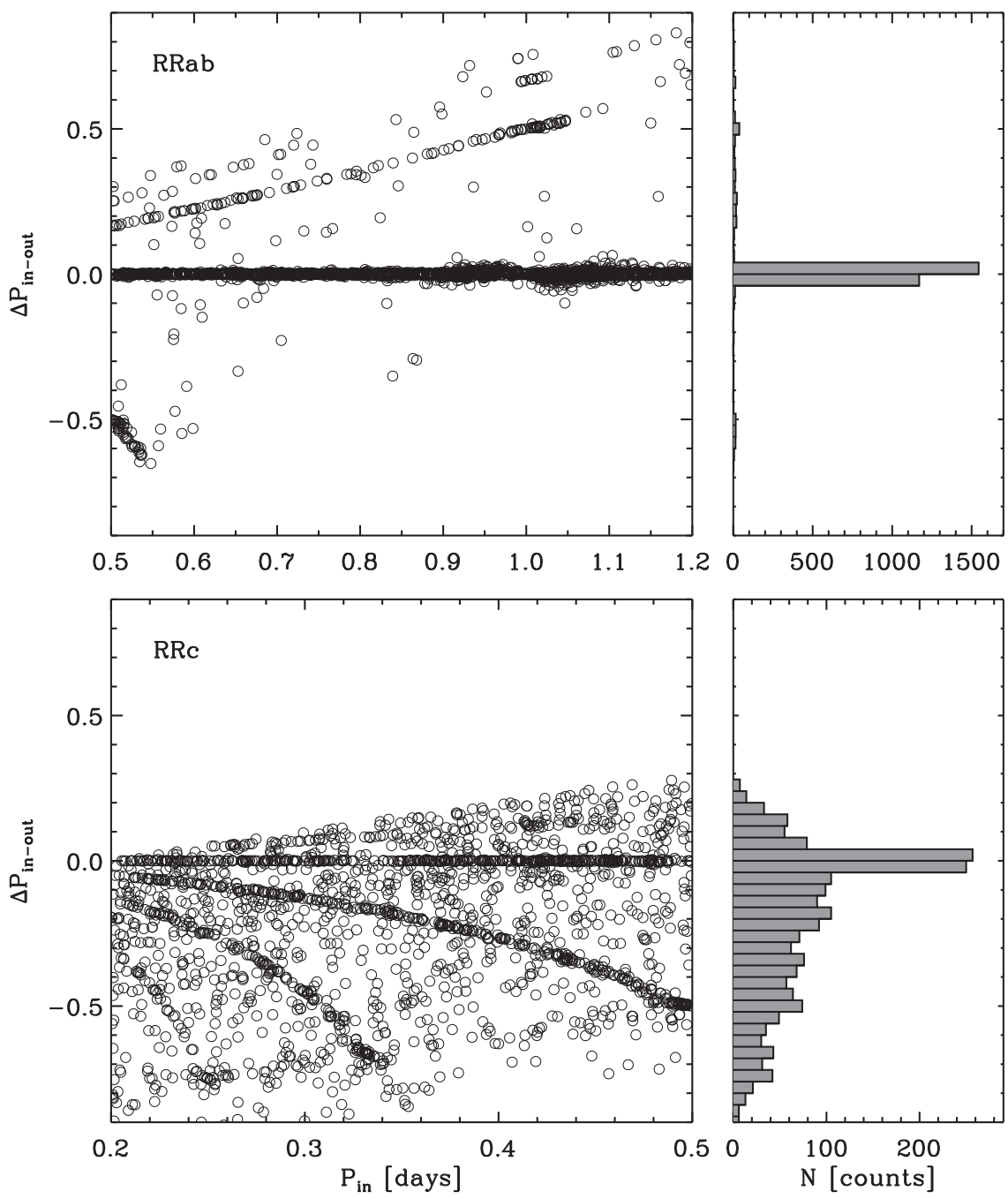

Figure 3. Our synthetic light curve simulations for Crater II, showing the differences between the input and output periods $\left(\Delta P_{\text {in-out }}\right)$ as a function of the input period (left panels) and their distributions (right panels), for the two different pulsation modes. The simulations indicate that $c$-type RR Lyrae stars are much more affected by aliasing compared to $a b$-type variables.

rest of the RR Lyrae stars in the CMD by its faint magnitude $(\langle V\rangle=21.334 \mathrm{mag})$, but it also has the shortest period $(P \simeq 0.235$ day), even though it is classified as $a b$-type from the shape of the light curve (see Figure 2 and Table 1). With this short period and pulsation mode, V97 might not be an RR Lyrae star, but rather a dwarf Cepheid (DC) (e.g., Mateo et al. 1998; Breger 2000; McNamara 2011) that probably belongs to the MW; it was therefore excluded from our analysis.

\subsection{Period-Amplitude Diagrams}

It is widely recognized that in contrast to the Galactic GCs, which present the well-known Oosterhoff dichotomy in the average period of RRab stars and their location in the periodamplitude (Bailey) diagram, the classical dSph galaxies have preferentially Oosterhoff-intermediate (Oo-int) properties (e.g., Catelan 2009; Clementini 2010; Smith et al. 2011, for reviews). Most RR Lyrae stars in the UFDs studied so far, on the other hand, are classified as Oo-int or Oosterhoff group II (Oo II) (e.g., Clementini 2014; Vivas et al. 2016, and references therein). Hence, a reliable Oosterhoff classification of Crater II based on the mean period of RRab stars and the Bailey diagram would help to understand the properties of its stellar population.
Figure 5 shows the period distribution and the Bailey diagram of the RR Lyrae stars in Crater II. We see in panel (a) that the RR Lyrae stars with the two different pulsation modes are well separated by their periods. The solid and dotted lines in panel (b) are the loci of Oosterhoff group I (Oo I) and Oo II clusters, respectively, according to the relation given by Zorotovic et al. (2010; see also Cacciari et al. 2005). The average periods of $83 a b$-type and $13 c$-type stars are $\left\langle P_{a b}\right\rangle=0.631 \pm 0.004$ day and $\left\langle P_{c}\right\rangle=0.411 \pm 0.009$ day, respectively, where the uncertainties are the standard errors of the means. If the 13 outliers (nine RRab and four RRc variables) are excluded, the mean periods of $74 a b$-type and nine $c$-type stars slightly change to $\left\langle P_{a b}\right\rangle=0.621 \pm 0.003$ day and $\left\langle P_{c}\right\rangle=0.423 \pm 0.004$ day, respectively. Note that these $\left\langle P_{a b}\right\rangle$ values are in the range of Oo II group clusters, over the Oosterhoff gap (0.58 day $\leqslant\left\langle P_{a b}\right\rangle \leqslant 0.62$ day, Catelan 2009). In the period-amplitude diagram, however, the Crater II RRab stars are located near the Oo I line, suggesting that Crater II may be classified as Oo I or Oo-int.

In Figure 6, we compare the RR Lyrae stars in Crater II with those in the Galactic halo field (data from Zinn et al. 2014), the other 14 UFDs (top panel), and the two classical dSphs, Draco and Carina (bottom panel), on the period-amplitude diagrams. 

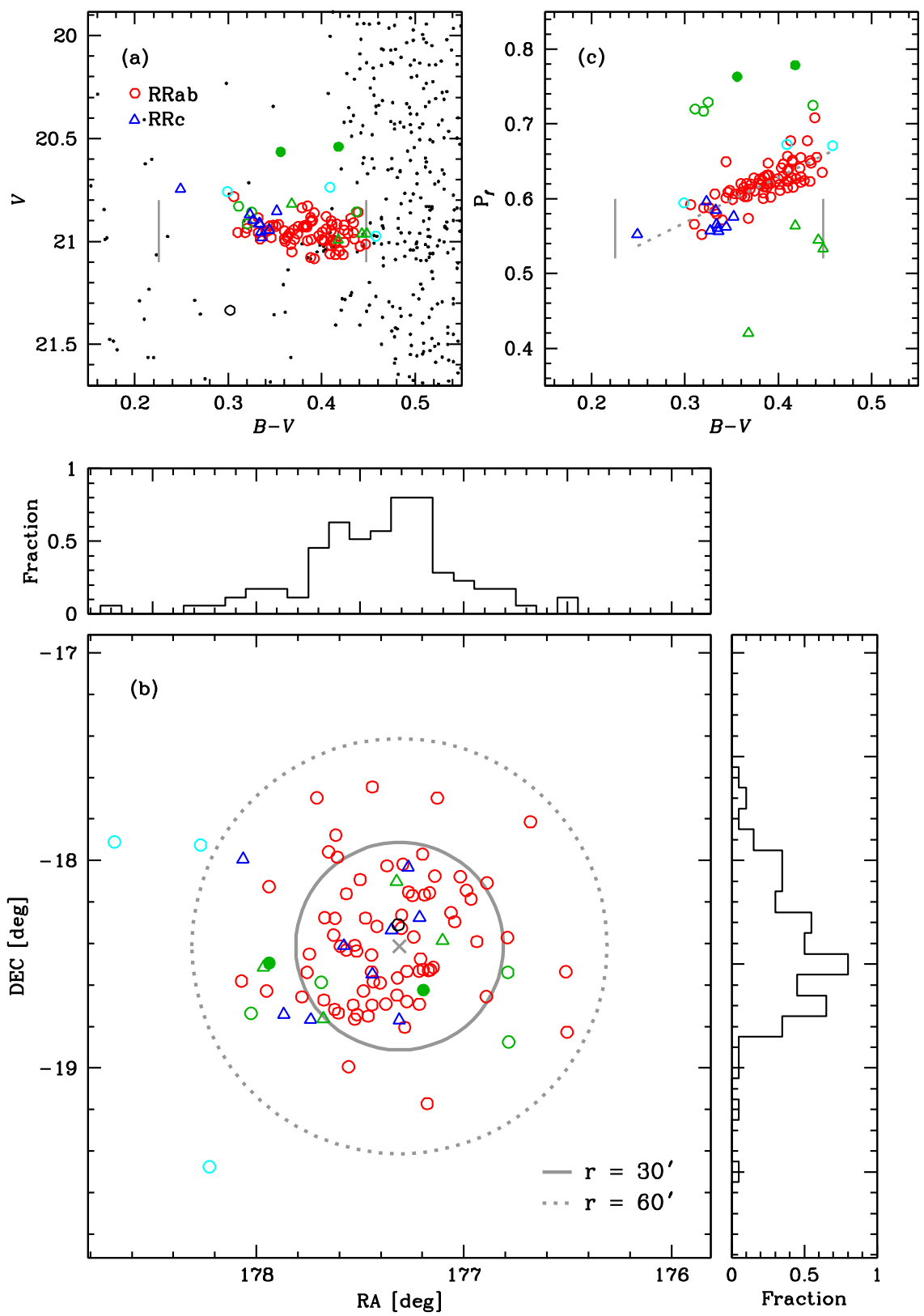

Figure 4. (a) CMD zoomed around the HB region, (b) spatial distribution, and (c) color-period diagram of the RR Lyrae stars, showing that most of them belong to Crater II. Circles and triangles denote $a b$ - and $c$-type variables, respectively. The two vertical gray lines in panels (a) and (c) represent the empirical instability strip estimated from the nine Galactic and LMC clusters in Walker (1998), which are also reddened by 0.05 mag (see the text). The three variables (cyan circles) outside $60^{\prime}$ from the center of the galaxy (gray cross) might be field RR Lyrae stars. The periods of RRc stars in panel (c) are fundamentalized assuming $P_{c} / P_{a b}=0.745$ (Nemec 1985; Clement et al. 2001). The dotted line in panel (c) is the robust linear fit to the data, where 10 RR Lyrae stars are outside the $3 \sigma$ range. The two brightest variables with the longest periods (green filled circles) might be either field RR Lyrae stars, highly evolved RR Lyrae stars, or ACs. The four RRab stars (green open circles) with long periods appear to be evolved RR Lyrae stars, while the four RRc stars (green triangles) with short periods are less certain. A probable non-RR Lyrae variable, V97, is marked as a black circle in panels (a) and (b).

Note that panel (a) is very similar to Figure 10 of Vivas et al. (2016), while the two brightest UFDs with $M_{V}<-7$, Canes Venatici I (CVn I) and Leo T, are included here too. ${ }^{7}$

$7 \mathrm{CVn}$ I is often considered to be a classical dSph rather than a UFD, because of its classical-dSph-like properties such as the total magnitude, broad RGB, Oo-int classification, half-light radius, and distribution of alpha-elements (e.g., Simon \& Geha 2007; Kuehn et al. 2008; Martin et al. 2008; Sand et al. 2012; Vargas et al. 2013). Leo T is probably not bound to the MW, located at a large heliocentric distance of $\sim 409 \mathrm{kpc}$ (Irwin et al. 2007; de Jong et al. 2008; Clementini et al. 2012; McConnachie 2012). Segue 1 is not included here. While one or two RR Lyrae stars are detected by Simon et al. (2011), their periods and amplitudes are not accurately measured (see also Vivas et al. 2016).
Data for RR Lyrae variables in the UFDs are taken from Kuehn et al. (2008, CVn I), Greco et al. (2008, Canes Venatici II, CVn II), Musella et al. (2009, Coma Berenices, ComBer), Siegel (2006, Boötes I; see also Dall'Ora et al. 2006), Vivas et al. (2016, Hydra II), Garofalo et al. (2013, Ursa Major I, UMa I), Moretti et al. (2009, Leo IV), Clementini et al. (2012, Leo T), and Boettcher et al. (2013, Segue II). For Boötes II, Boötes III (Sesar et al. 2014), and Ursa Major II (UMa II) (Dall'Ora et al. 2012), we used the revised data by Vivas et al. (2016). For Boötes I, the $A_{B}$ values were converted to $A_{V}$ using equations derived by Dorfi \& Feuchtinger (1999) in the same way as Vivas et al. (2016). In the case of Hercules, we added 

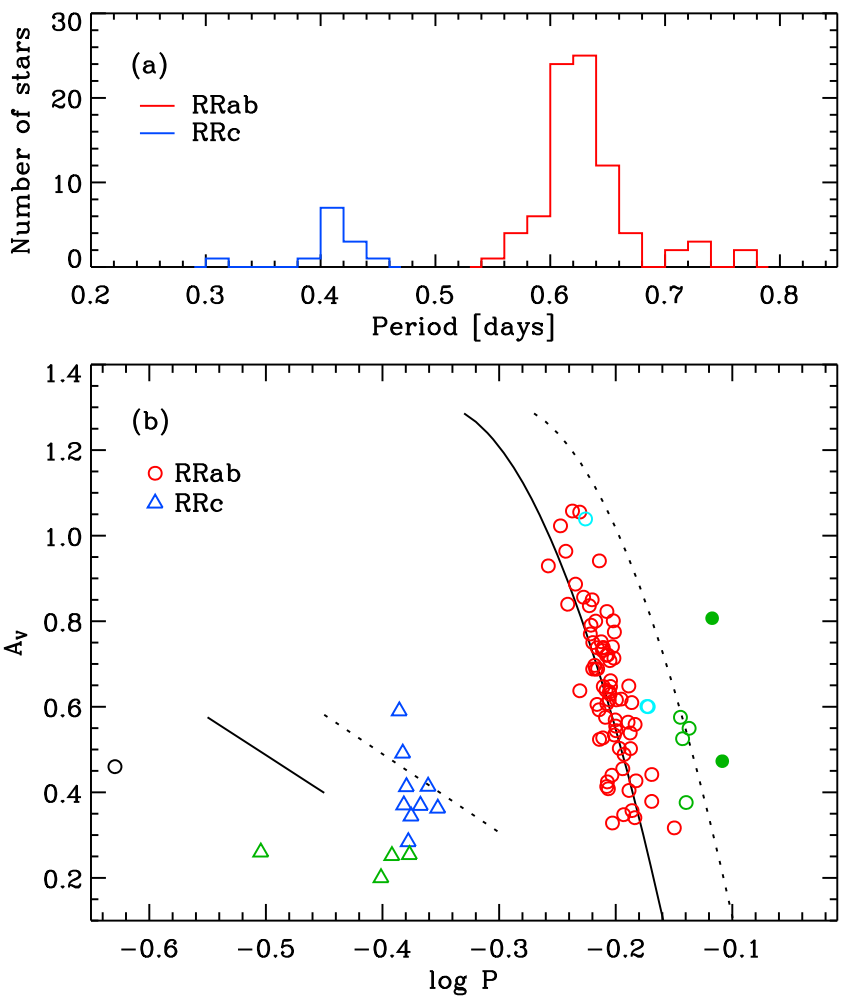

Figure 5. (a) Period distribution and (b) period-amplitude diagram of the RR Lyrae stars in Crater II. The solid and dotted lines in panel (b) represent the loci of the MW Oo I and Oo II GCs, respectively (Cacciari et al. 2005; Zorotovic et al. 2010). Symbols are the same as in Figure 4.

three (one RRab and two RRc) stars recently identified by Garling et al. (2018) to the data of Musella et al. (2012), where the SDSS $g$-band amplitudes, $A_{g}$, of these three variables were transformed to $A_{V}$, using the equation, $V=g-0.59$ $(g-r)-0.01$, by Jester et al. (2005, see their Table 1). Similarly, without color information, the $A_{g}$ values of three RRab stars in Leo V (Medina et al. 2017), were converted to $A_{V}$, by assuming $(g-r)=0.15$ as a rough estimate for the mean color of the RRab stars (An et al. 2008).

As presented in panel (a), the RRab stars in the UFDs (blue symbols), except for Crater II and CVn I, are broadly scattered in the Oo-int and Oo II regions, while most of those $(\sim 73 \%)$ in the Galactic halo field belong to Oo I (Zinn et al. 2014) with some hints of the Oosterhoff dichotomy. Crater II and CVn I, on the other hand, are clearly distinct from the other UFDs, but rather similar to the two classical dSphs with Oo-int properties, Draco (data from Kinemuchi et al. 2008) and Carina (data from Coppola et al. 2015), shown in panel (b). It is notable that, not only the distribution of RRab stars in the Bailey diagram, but also the mean period of RRab stars $\left(\left\langle P_{a b}\right\rangle=0.631\right.$ day $)$, and the $c$-type fraction $(N(c) / N(a b+c) \simeq 0.14)$ of Crater II are comparable to those of CVn I and the two classical dSphs, respectively, i.e., $\left\langle P_{a b}\right\rangle=0.60$ day and $N(c) / N(a b+c)=0.22$ for CVn I, $\left\langle P_{a b}\right\rangle=0.615$ day and $N(c+d) / N(a b+c+d)=$ 0.21 for Draco, and $\left\langle P_{a b}\right\rangle=0.634$ day and $N(c+d) / N(a b+$ $c+d)=0.23$ for Carina, where we included Blazhko RRab stars and double-mode ( $d$-type) pulsators (see also Stetson et al. 2014; Baker \& Willman 2015, for the several other dSphs). These similarities undoubtedly suggest an Oo-int classification for Crater II, even though it has a somewhat long $\left\langle P_{a b}\right\rangle$, which corresponds to the Oo II group. This also leads us to conclude that, based on the RR Lyrae properties, Crater II is more like a classical dSph rather than a UFD.

\subsection{Metallicity}

RR Lyrae stars can further be used to estimate metallicity, reddening, and distance of a stellar system independently of other methods, since their pulsation properties (including period and amplitude) are correlated with the stellar evolution parameters such as mass, metallicity, temperature, and luminosity (see, e.g., van Albada \& Baker 1971; Sandage 1993, 2006; Di Criscienzo et al. 2004; Bono et al. 2007; Jeffery et al. 2011). To obtain metallicities for the individual RRab stars of Crater II, we used the empirical period-amplitude-metallicity relation derived by Alcock et al. (2000),

$$
[\mathrm{Fe} / \mathrm{H}]=-8.85\left(\log P_{a b}+0.15 A_{V}\right)-2.60,
$$

where $[\mathrm{Fe} / \mathrm{H}]$ is on the Zinn \& West (1984) scale with an accuracy of $\sigma_{[\mathrm{Fe} / \mathrm{H}]}=0.31 \mathrm{dex}$. The upper panel of Figure 7 shows the metallicity distribution of the RRab stars calculated from this equation and a Gaussian fit to the histogram, which gives the mean metallicity, $\langle[\mathrm{Fe} / \mathrm{H}]\rangle=-1.65 \pm 0.15(0.02)$. The uncertainty is the standard deviation and the number in parentheses presents the standard error of the mean.

This metallicity is in good agreement with the estimation from the isochrone fitting $([\mathrm{Fe} / \mathrm{H}]=-1.7 \pm 0.1)$ by Torrealba et al. (2016a), but somewhat more metal-rich than the value from the spectroscopic measurement $(\langle[\mathrm{Fe} / \mathrm{H}]\rangle=-1.98 \pm 0.1)$ by Caldwell et al. (2017). Note, however, that as Caldwell et al. (2017) already stated, their estimation might be systematically metal-poor, probably due to the zero-point uncertainty of metallicity. While the relation of Alcock et al. (2000), which we adopt here, is more reliable than the other methods based only on the mean period of RRab stars (e.g., Sandage 2006; Sarajedini et al. 2006), it should be noted that the derived $[\mathrm{Fe} / \mathrm{H}]$ value has also intrinsically a large dispersion and uncertainty. For example, it can be affected by the luminosity (evolution) effect on the period of RRab stars (Yang et al. 2010) and/or the selection of calibration cluster (Bono et al. 2007; Jeffery et al. 2011).

\subsection{Reddening and Distance}

Sturch (1966) has shown that $a b$-type RR Lyrae stars have nearly identical intrinsic colors, $(B-V)_{0}$, in the phase interval from 0.5 to 0.8 (i.e., at minimum light), only weakly correlated with period and metallic-line blanketing. By combining the Sturch's formula with the calibration between metallicity and line blanketing effect from Butler (1975), Walker (1990) presented the following relation for the interstellar extinction:

$$
\begin{aligned}
E(B-V)= & (B-V)_{\min }-0.24 P_{a b} \\
& -0.056[\mathrm{Fe} / \mathrm{H}]-0.336,
\end{aligned}
$$

where $(B-V)_{\min }$ is the average color at the minimum light (phase between 0.5 and 0.8 ) and the metallicity scale is that of Zinn \& West (1984). Adopting the metallicities from the previous subsection, we obtain reddening values of the individual RRab stars. The distribution of $E(B-V)$ values and a Gaussian fit at the histogram are plotted (black solid lines) in panel (b) of Figure 7, which yields $\langle E(B-V)\rangle=0.09 \pm 0.02$ (0.003), where the uncertainty is the standard deviation and the value in 

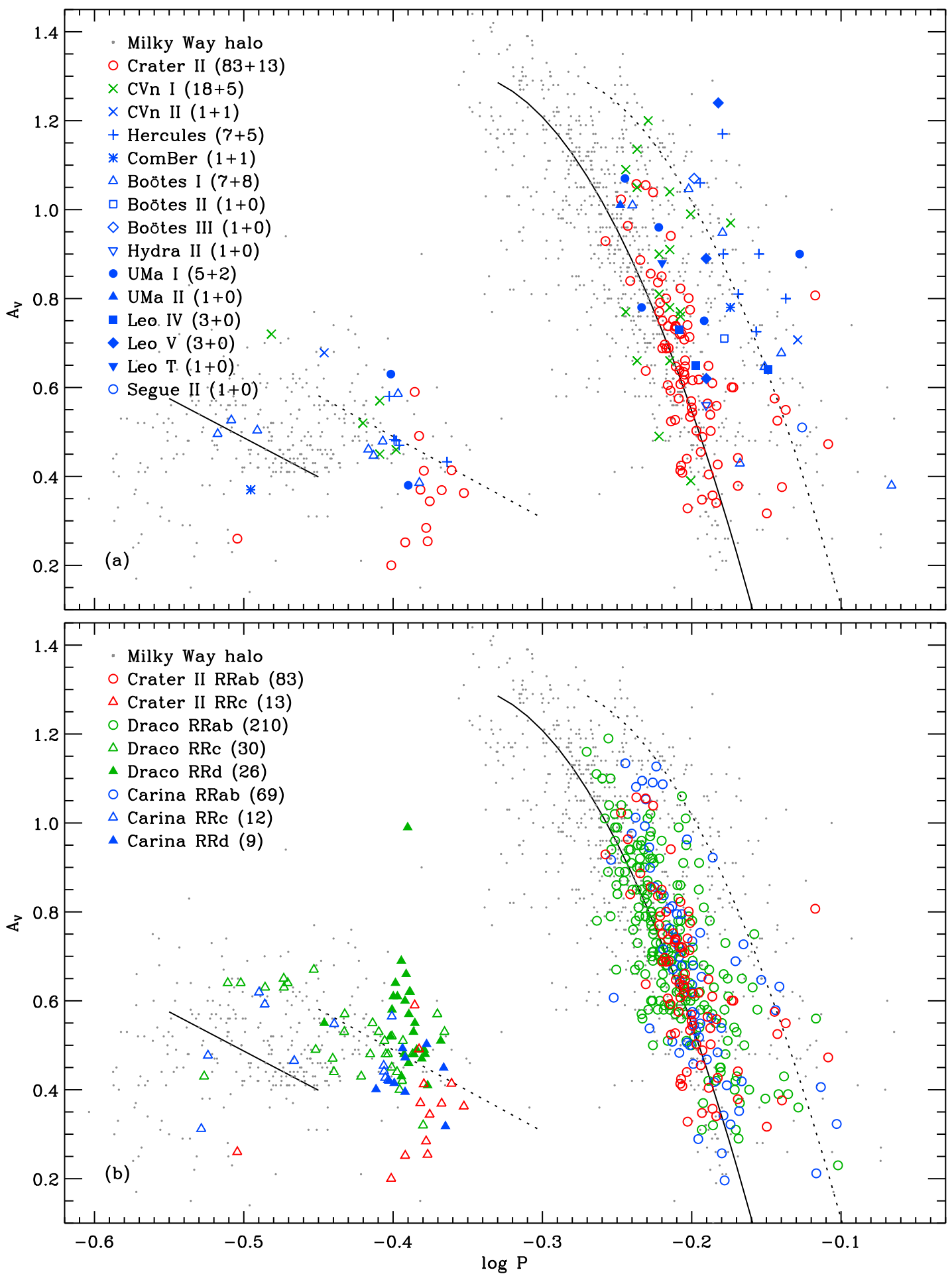

Figure 6. (a) Same as Figure 5(b), but here the RR Lyrae stars in Crater II (red circles) are compared with those in the MW halo (gray dots), CVn I (green crosses), and the other 13 UFDs (blue symbols). Note that this is just an update of Figure 10 in Vivas et al. (2016). Numbers in parentheses are the numbers of $a b$ - and $c$-type variables separated by plus signs. (b) Same as panel (a), but compared with those in the two classical dwarfs, Draco (green) and Carina (blue). Numbers in parentheses are the numbers of variables.

parentheses is the standard error of the mean. As already noted by Walker (1998; see also Piersimoni et al. 2002; Clementini et al. 2003; Musella et al. 2012), the Sturch's method tends to overestimate the extinction by $0.02-0.03 \mathrm{mag}$. If this effect is taken into account, $\langle E(B-V)\rangle$ would be reduced to $\sim 0.06$. This corrected value is, however, still larger than the estimation based on the Schlegel et al. (1998) maps, from which we obtained $\langle E(B-V)\rangle=0.034$ for $r<30^{\prime}$ and $\langle E(B-V)\rangle=$ 0.037 for $r<60^{\prime}$ areas around the center of Crater II (see solid and dotted gray circles in Figure 4(b)).

Another independent estimation for the interstellar reddening can be made using the $B$-band amplitude $\left(A_{B}\right)$, metallicity, and period of RRab stars, namely, the amplitude-color-metallicity (ACZ) and/or period-amplitude-color-metallicity (PACZ) 

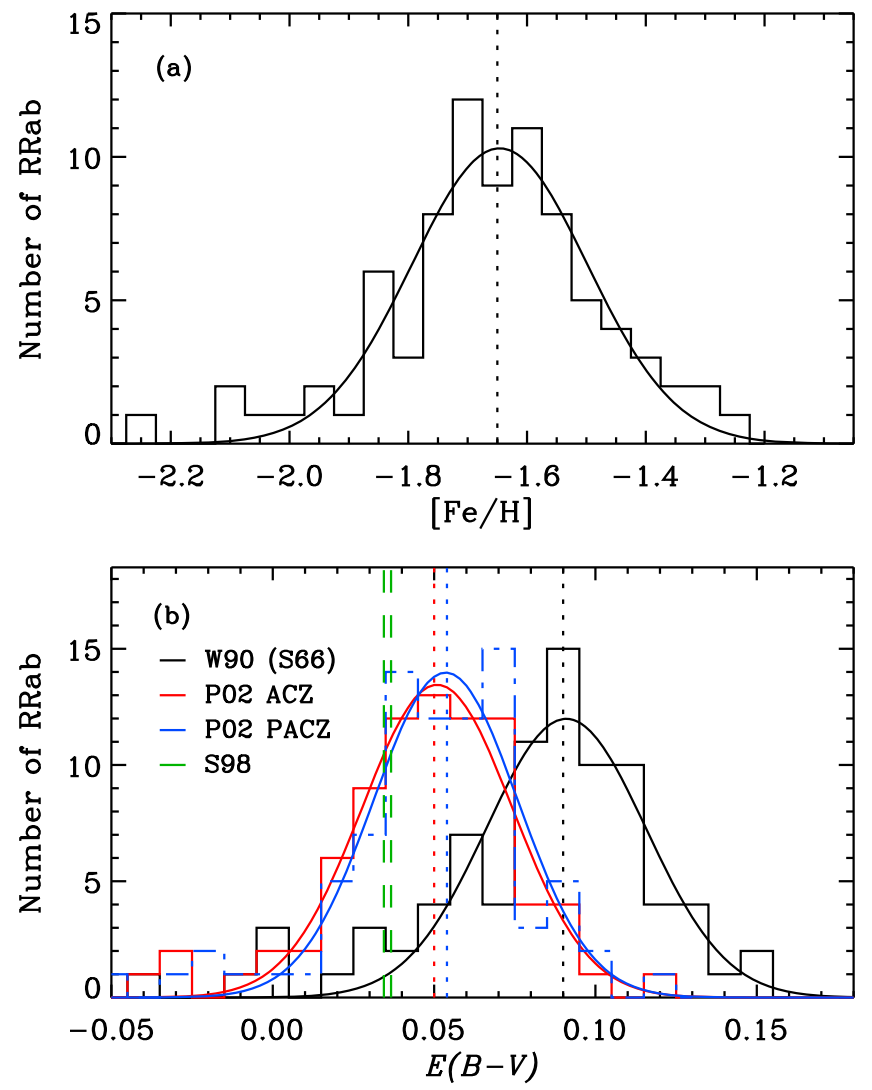

Figure 7. (a) Metallicity distribution of individual RRab stars derived from the equation of Alcock et al. (2000) and a Gaussian fit (solid curve). (b) Reddening distributions of RRab stars obtained from the equations of Walker (1990, W90, based on Sturch 1966, S66) and Piersimoni et al. (2002, P02, ACZ and PACZ) and Gaussian fits to the data (solid curves). Note that the Sturch's method tends to overestimate the reddening by $\sim 0.3 \mathrm{mag}$ (see the text). The two dashed green lines denote the mean reddenings from the map of Schlegel et al. (1998, S98) for $r<30^{\prime}$ and $r<60^{\prime}$ regions around the center of the galaxy. Dotted lines indicate the peaks of the distributions. The peak values of metallicity and reddenings hardly change, even if the nine $a b$-type outliers (i.e., the three stars outside $2 r_{h}$ and the six stars with long periods) are excluded.

relations derived by Piersimoni et al. (2002),

$$
\begin{aligned}
(B-V)_{0}= & 0.448-0.078 A_{B}+0.012[\mathrm{Fe} / \mathrm{H}], \\
(B-V)_{0}= & 0.507-0.052 A_{B}+0.223 \log P_{a b} \\
& +0.036[\mathrm{Fe} / \mathrm{H}] .
\end{aligned}
$$

The $E(B-V)$ values are then calculated by subtracting these intrinsic colors, $(B-V)_{0}$, from the magnitude-mean colors, $(B-V)_{m}$. The histograms of reddening values obtained from these two relations and Gaussian fits for them are also plotted in panel (b) in Figure 7 (red and blue colors, respectively). The peaks of the distributions give $\langle E(B-V)\rangle=0.05 \pm 0.02$ (0.003) for both ACZ and PACZ relations, where the uncertainty is the standard deviation with the standard error of the mean in parentheses. This is roughly in the middle of the values obtained from Sturch's method (when shifted by -0.03 mag) and the Schlegel et al. (1998) maps, and therefore we adopt this as our final estimate for the reddening (see Table 2).

Once metallicity and reddening are determined, we can estimate the distance to the galaxy from the luminositymetallicity relation of RR Lyrae stars. There have been many attempts to establish a universal $M_{V}(\mathrm{RR})-[\mathrm{Fe} / \mathrm{H}]$ relation, but some discrepancies still remain between the methods (see, e.g.,
Table 2

Metallicity, Reddening, and Distance of Crater II Derived from the RRab Stars

\begin{tabular}{lc}
\hline \hline Properties & Values \\
\hline Metallicity, $\langle[\mathrm{Fe} / \mathrm{H}]\rangle$ & $-1.65 \pm 0.15(0.02)^{\mathrm{a}}$ \\
Reddening, $\langle E(B-V)\rangle$ & $0.05 \pm 0.02(0.003)^{\mathrm{a}}$ \\
Distance modulus, $(m-M)_{0}(\mathrm{mag})$ & $20.25 \pm 0.10$ \\
Distance, $d_{\odot}(\mathrm{kpc})$ & $112 \pm 5$ \\
\hline
\end{tabular}

Note.

${ }^{a}$ The uncertainty is the standard deviation and the number in parentheses is the standard error of the mean.

Chaboyer 1999; Cacciari \& Clementini 2003; Catelan 2009, for reviews). We adopt here the slope of the relation, $\Delta M_{V}(\mathrm{RR}) / \Delta$ $[\mathrm{Fe} / \mathrm{H}]=0.23( \pm 0.04)$, and the zero point, $M_{V}=0.56( \pm 0.12)$ at $[\mathrm{Fe} / \mathrm{H}]=-1.6$, from Chaboyer (1999, see Equation (8) in that paper). The relation is then

$$
M_{V}(\mathrm{RR})=0.23[\mathrm{Fe} / \mathrm{H}]+0.93,
$$

and yields the absolute magnitude $M_{V}(\mathrm{RR})=0.55 \pm 0.07$, by applying the mean metallicity of RRab stars derived above, $\langle[\mathrm{Fe} / \mathrm{H}]\rangle=-1.65 \pm 0.15(0.02)$. The uncertainty in $M_{V}$ was propagated from the intrinsic deviation of the Alcock et al. (2000) relation.

Combining this with the reddening estimated above and the mean apparent $V$-magnitude, $\left\langle V_{\mathrm{RR}}\right\rangle=20.95 \pm 0.01$, we finally obtain a distance modulus of $(m-M)_{0}=20.25 \pm 0.10$ and a distance of $d_{\odot}=112 \pm 5 \mathrm{kpc}$ to Crater II (see Table 2). The uncertainty of $(m-M)_{0}$ is the quadratic sum of the errors in the $M_{V}(\mathrm{RR})$ and the $V$-band extinction, $\sim 3.1 E(B-V)$. Note that this distance estimate agrees with the suggestion by Torrealba $\left(2016 \mathrm{a}, d_{\odot}=117.5 \pm 1.1 \mathrm{kpc}\right)$ within the uncertainty.

\section{Discussion}

Using the $1.6 \mathrm{~m}$ wide-field KMTNet-CTIO telescope, we performed time-series $B, V$ photometry of Crater II, one of the largest and lowest surface brightness dwarf satellites of the MW. We have detected and characterized 96 RR Lyrae stars (83 RRab and $13 \mathrm{RRc)}$ with the template light curve fitting routine, RRFIT, by Yang \& Sarajedini (2012) and Yang et al. $(2010,2014)$. The mean period of RRab stars, $\left\langle P_{a b}\right\rangle=0.631 \pm$ 0.004 day, is somewhat longer than the Oosterhoff gap, but the $c$-type fraction is low $(\sim 0.14)$ and the location of $a b$-type variables in the period-amplitude diagram is close to the locus of Oo I group clusters. In terms of these Oosterhoff properties, Crater II is very similar to CVn I and the two classical dSph, Draco and Carina. This not only suggests an Oo-int classification for this galaxy, but also leads us to conclude that it can be categorized as a classical dSph rather than a UFD.

These similarities in RR Lyrae stars between Crater II and the classical dSphs indicate that the RR Lyrae properties are independent of the surface brightness of the galaxy. Instead, given the fact that Crater II and CVn I, with $M_{V} \approx-8.2$ and -8.6 , respectively, are much more luminous than typical UFDs and are comparable to the faint classical dSphs, like Draco and Carina with $M_{V} \approx-8.8$ and $-9.3,{ }^{8}$ respectively

\footnotetext{
8 Unlike the other dwarfs considered here, Carina has a sizable amount of intermediate-age population with a red HB that cannot produce RR Lyrae stars (Monelli et al. 2003). If we assume that roughly half of the population in Carina are old enough to host RR Lyrae stars, then the total magnitude of the old population only would be $M_{V} \simeq-8.5$.
} 


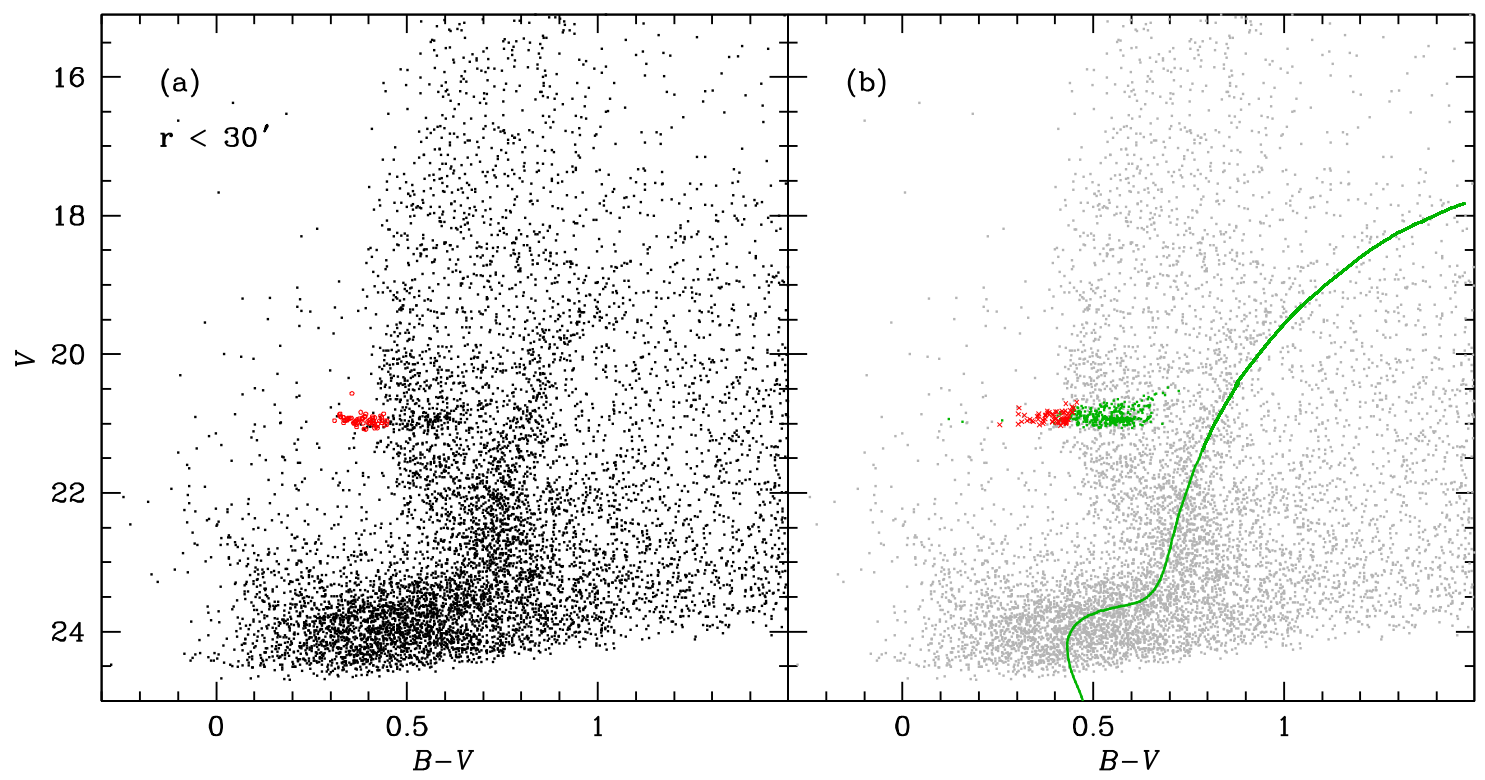

Figure 8. Comparison of our population models with the observations of Crater II. (a) Observed CMD, similar to Figure 1(a) with red open circles for the RR Lyrae stars. (b) Our population models (green lines and points) on the observed CMD (gray points). Red crosses denote the model RR Lyrae stars. Parameters used in our simulation are listed in Table 3. Adopted reddening and distance modulus are $E(B-V)=0.05$ and $(m-M)_{0}=20.30$ mag, where $(m-M)_{0}$ was slightly revised by $+0.05 \mathrm{mag}$ (see Table 2).

(Martin et al. 2008; McConnachie 2012; Sand et al. 2012; Torrealba et al. 2016a), the RR Lyrae properties appear to be more related to the total luminosity of the galaxy. This interpretation also agrees well with the luminosity-metallicity (or mass-metallicity) relation of (dwarf) galaxies (e.g., Grebel et al. 2003; Kirby et al. 2008; Willman \& Strader 2012; Conn et al. 2018; see also Yang et al. 2014, for a recent compilation of nearby dwarfs), in the sense that stellar population properties are correlated with the luminosity (stellar mass) of the system.

We have then constructed stellar population models, to investigate the star formation history of Crater II, following the techniques outlined by Lee et al. $(1990,1994)$ and Joo \& Lee (2013). We used Yonsei-Yale $\left(\mathrm{Y}^{2}\right)$ isochrones and HB evolutionary tracks (Yi et al. 2008; Han et al. 2009a), and employed the stellar model atmospheres by Castelli \& Kurucz (2003) for temperature-color transformations. Readers are referred to Joo \& Lee (2013) and references therein for details of the model construction. In Figure 8, our synthetic model is compared with the observed CMDs for $r<30^{\prime}$.

In this model, we first adopted the metallicity, reddening, and distance modulus from the RR Lyrae stars, under the assumptions of $[\alpha / \mathrm{Fe}]=0.3$ and the standard helium-enrichment parameter $(\Delta Y / \Delta Z=2.0, Y=0.23+Z(\Delta Y / \Delta Z))$. The age value was then adjusted until the best match between the model and the observed CMD was obtained, while those parameters from RR Lyrae stars were mostly fixed. The input parameters used in our best simulation are listed in Table 3. Figure 8 shows that there is no clear sign of young stellar populations, in addition to the old stellar population reproduced by our model in panel (b), indicating that Crater II is dominated by an old stellar population. The age obtained $(\sim 10.5 \mathrm{Gyr})$ is somewhat younger than our previous estimation of the old populations in the MW GCs (Yoon et al. 2008; Joo \& Lee 2013) and the determination of UFDs by Brown et al. (2012, 2014). This is easily understood by the red HB morphology of Crater II despite its low metallicity.
A crucial question regarding present-day dwarf galaxies is whether they are surviving counterparts of the building blocks that merged to form larger galaxies, as predicted by the $\Lambda \mathrm{CDM}$ hierarchical clustering paradigm. Recent analyses based on the RR Lyrae properties suggest that galaxies, like most dwarf companions of the MW are probably not the major contributor to the Galactic halo, except the most massive ones. For instance, Zinn et al. (2014) proposed that the preponderance of Oo I variables in the MW halo, shown in the period-amplitude diagram, may be explained by accretion of galaxies resembling the massive MW satellites such as LMC, SMC, Fornax, and Sgr, while minority Oo-int and Oo II stars may partially come from the systems like low-mass dSphs or UFDs. Stetson et al. (2014) and Fiorentino et al. (2015) found that, contrary to the MW halo and GCs, there is a complete lack of high-amplitude short-period (HASP) RRab stars in most dwarf galaxies. Fiorentino et al. (2015) have further shown that those stars are only present in the metal-rich systems $([\mathrm{Fe} / \mathrm{H}] \gtrsim-1.5)$, and concluded that only massive dwarf galaxies with a broad metallicity distribution like LMC and Sgr may be primary building block candidates of the MW halo, with limited contribution from less massive systems like present-day dSphs and UFDs.

In this context, with a lack of the HASP RRab stars (as shown in Figures 5 and 6) and relatively low stellar mass, Crater II also may not be directly related to the MW building blocks. Given its size and surface brightness, however, the presence of Crater II itself implies that there might be even larger galaxies yet to be discovered, under the current surface brightness detection limit, as possible massive (and therefore metal-rich) remnants of the building blocks. Further studies of dwarf galaxies including a more complete census and variable star observations will be important to better understand the role of dwarf galaxies in the assembly of present-day massive galaxies. 
Table 3

Input Parameters Used in Our Best Simulation of the Crater II Stellar Population

\begin{tabular}{lccccc}
\hline \hline$Z$ & {$[\mathrm{Fe} / \mathrm{H}]^{\mathrm{a}}$} & $Y^{\mathrm{b}}$ & $\begin{array}{c}\text { Age } \\
(\mathrm{Gyr})\end{array}$ & $\begin{array}{c}\text { Mass-loss }^{\mathrm{c}} \\
\left(M_{\odot}\right)\end{array}$ & $\begin{array}{c}\sigma_{M}{ }^{\mathrm{d}} \\
\left(M_{\odot}\right)\end{array}$ \\
\hline 0.00071 & -1.65 & 0.231 & 10.5 & 0.187 & 0.02 \\
\hline
\end{tabular}

Notes.

${ }^{\mathrm{a}}[\alpha / \mathrm{Fe}]=0.3$

${ }^{\mathrm{b}}$ From the standard helium-enrichment parameter, i.e., $\Delta Y / \Delta Z=2.0, Y=$ $0.23+Z(\Delta Y / \Delta Z)$.

${ }^{c}$ Mean mass-loss on the RGB for the Reimers (1977) mass-loss parameter, $\eta=0.53$.

${ }^{\mathrm{d}}$ Mass dispersion on the $\mathrm{HB}$.

We thank the anonymous referee for a number of helpful suggestions. This research has made use of the KMTNet system operated by the Korea Astronomy and Space Science Institute (KASI), and the data were obtained at one of three host sites, CTIO in Chile. H. Jeong acknowledges support from the Basic Science Research Program through the National Research Foundation (NRF) of Korea, funded by the Ministry of Education (NRF-2013R1A6A3A04064993). S.C.R. was partially supported by the Basic Science Research Program through the NRF of Korea funded by the Ministry of Education (2018R1A2B2006445). Support for this work was also provided by the NRF to the Center for Galaxy Evolution Research (2017R1A5A1070354). H. Jerjen acknowledges the support of the Australian Research Council through Discovery Project DP150100862. This research was made possible through the use of the APASS, funded by the Robert Martin Ayers Sciences Fund.

\section{ORCID iDs}

Helmut Jerjen (1) https://orcid.org/0000-0003-4624-9592

\section{References}

Alcock, C., Allsman, R. A., Alves, D. R., et al. 2000, AJ, 119, 2194 An, D., Johnson, J. A., Clem, J. L., et al. 2008, ApJS, 179, 326 Bahl, H., \& Baumgardt, H. 2014, MNRAS, 438, 2916 Baker, M., \& Willman, B. 2015, AJ, 150, 160

Bechtol, K., Drlica-Wagner, A., Balbinot, E., et al. 2015, ApJ, 807, 50 Belokurov, V. 2013, NewAR, 57, 100

Belokurov, V., Walker, M. G., Evans, N. W., et al. 2008, ApJL, 686, L83 Belokurov, V., Walker, M. G., Evans, N. W., et al. 2009, MNRAS, 397, 1748 Belokurov, V., Walker, M. G., Evans, N. W., et al. 2010, ApJL, 712, L103 Belokurov, V., Zucker, D. B., Evans, N. W., et al. 2006, ApJL, 647, L111 Belokurov, V., Zucker, D. B., Evans, N. W., et al. 2007, ApJ, 654, 897 Bertin, E. 2006, in ASP Conf. Ser. 351, Astronomical Data Analysis Software and Systems XV, ed. C. Gabriel et al. (San Francisco, CA: ASP), 112

Bertin, E., Mellier, Y., Radovich, M., et al. 2002, in ASP Conf. Ser. 281, Astronomical Data Analysis Software and Systems XI, ed. D. A. Bohlender, D. Durand, \& T. H. Handley (San Francisco, CA: ASP), 228

Boettcher, E., Willman, B., Fadely, R., et al. 2013, AJ, 146, 94

Bono, G., Caputo, F., \& Di Criscienzo, M. 2007, A\&A, 476, 779

Breger, M. 2000, in ASP Conf. Ser. 210, Delta Scuti and Related Stars, ed. M. Breger \& M. Montgomery (San Francisco, CA: ASP), 3

Brown, T. M., Tumlinson, J., Geha, M., et al. 2012, ApJL, 753, L21

Brown, T. M., Tumlinson, J., Geha, M., et al. 2014, ApJ, 796, 91

Bullock, J. S., \& Johnston, K. V. 2005, ApJ, 635, 931

Butler, D. 1975, ApJ, 200, 68

Cacciari, C., \& Clementini, G. 2003, in Stellar Candles for the Extragalactic Distance Scale, Vol. 635, ed. D. Alloin \& W. Gieren (Berlin: Springer), 105 Cacciari, C., Corwin, T. M., \& Carney, B. W. 2005, AJ, 129, 267 Caldwell, N., Walker, M. G., Mateo, M., et al. 2017, ApJ, 839, 20
Castelli, F., \& Kurucz, R. L. 2003, in IAU Symp. 210, Modelling of Stellar Atmospheres, ed. N. Piskunov, W. W. Weiss, \& D. F. Gray (San Francisco, CA: ASP), 20

Catelan, M. 2009, Ap\&SS, 320, 261

Chaboyer, B. 1999, in Post-Hipparcos Cosmic Candles, Vol. 237 ed. A. Heck \& F. Caputo (Dordrecht: Kluwer), 111

Chambers, K. C., Magnier, E. A., Metcalfe, N., et al. 2016, arXiv:1612.05560

Clement, C. M., Muzzin, A., Dufton, Q., et al. 2001, AJ, 122, 2587

Clementini, G. 2010, in Variable Stars, the Galactic Halo and Galaxy Formation, ed. N. Samus, C. Sterken, \& L. Szabados (Moscow: Sternberg Astronomical Institute of Moscow Univ.), 107

Clementini, G. 2014, in Proc. IAU Symp. 301, Precision Asteroseismology, ed. J. A. Guzik et al. (Cambridge: Cambridge Univ. Press), 129

Clementini, G., Cignoni, M., Contreras Ramos, R., et al. 2012, ApJ, 756, 108

Clementini, G., Gratton, R., Bragaglia, A., et al. 2003, AJ, 125, 1309

Conn, B. C., Jerjen, H., Kim, D., \& Schirmer, M. 2018, ApJ, 852, 68 Coppola, G., Marconi, M., Stetson, P. B., et al. 2015, ApJ, 814, 71 Dall'Ora, M., Clementini, G., Kinemuchi, K., et al. 2006, ApJL, 653, L109 Dall'Ora, M., Kinemuchi, K., Ripepi, V., et al. 2012, ApJ, 752, 42 de Jong, J. T. A., Harris, J., Coleman, M. G., et al. 2008, ApJ, 680, 1112 DES Collaboration 2016, MNRAS, 460, 1270

Di Criscienzo, M., Marconi, M., \& Caputo, F. 2004, ApJ, 612, 1092

Dorfi, E. A., \& Feuchtinger, M. U. 1999, A\&A, 348, 815

Drlica-Wagner, A., Bechtol, K., Allam, S., et al. 2016, ApJL, 833, L5

Drlica-Wagner, A., Bechtol, K., Rykoff, E. S., et al. 2015, ApJ, 813, 109

Fiorentino, G., Bono, G., Monelli, M., et al. 2015, ApJL, 798, L12

Frebel, A., Simon, J. D., Geha, M., \& Willman, B. 2010, ApJ, 708, 560

Garling, C., Willman, B., Sand, D. J., et al. 2018, ApJ, 852, 44

Garofalo, A., Cusano, F., Clementini, G., et al. 2013, ApJ, 767, 62

Grebel, E. K., Gallagher, J. S., III, \& Harbeck, D. 2003, AJ, 125, 1926

Greco, C., Dall'Ora, M., Clementini, G., et al. 2008, ApJL, 675, L73

Grillmair, C. J. 2006, ApJL, 645, L37

Grillmair, C. J. 2009, ApJ, 693, 1118

Han, S.-I., Kim, Y.-C., Lee, Y.-W., et al. 2009a, in ESO Astrophysics Symposia, Globular Clusters-Guides to Galaxies, ed. T. Richtler \& S. Larsen (Berlin: Springer), 33

Han, S.-I., Lee, Y.-W., Joo, S.-J., et al. 2009b, ApJL, 707, L190

Han, S.-I., Lim, D., Seo, H., \& Lee, Y.-W. 2015, ApJL, 813, L43

Irwin, M. J., Belokurov, V., Evans, N. W., et al. 2007, ApJL, 656, L13

Jeffery, E. J., Smith, E., Brown, T. M., et al. 2011, AJ, 141, 171

Jerjen, H. 2010, AdAst, 2010, 434390

Jester, S., Schneider, D. P., Richards, G. T., et al. 2005, AJ, 130, 873

Joo, S.-J., \& Lee, Y.-W. 2013, ApJ, 762, 36

Kauffmann, G., White, S. D. M., \& Guiderdoni, B. 1993, MNRAS, 264, 201

Kim, D., \& Jerjen, H. 2015, ApJL, 808, L39

Kim, D., Jerjen, H., Geha, M., et al. 2016a, ApJ, 833, 16

Kim, D., Jerjen, H., Mackey, D., Da Costa, G. S., \& Milone, A. P. 2015a, ApJL, 804, L44

Kim, D., Jerjen, H., Milone, A. P., Mackey, D., \& Da Costa, G. S. 2015b, ApJ, 803, 63

Kim, H.-S., Yoon, S.-J., Sohn, S. T., et al. 2013, ApJ, 763, 40

Kim, S.-L., Lee, C.-U., Park, B.-G., et al. 2016b, JKAS, 49, 37

Kinemuchi, K., Harris, H. C., Smith, H. A., et al. 2008, AJ, 136, 1921

Kirby, E. N., Simon, J. D., Geha, M., Guhathakurta, P., \& Frebel, A. 2008, ApJL, 685, L43

Klypin, A., Kravtsov, A. V., Valenzuela, O., \& Prada, F. 1999, ApJ, 522, 82

Koch, A., \& Rich, R. M. 2014, ApJ, 794, 89

Koposov, S., Belokurov, V., Evans, N. W., et al. 2008, ApJ, 686, 279

Koposov, S. E., Belokurov, V., Torrealba, G., \& Evans, N. W. 2015, ApJ, 805,130

Koposov, S. E., Yoo, J., Rix, H.-W., et al. 2009, ApJ, 696, 2179

Kovács, G., \& Kupi, G. 2007, A\&A, 462, 1007

Kuehn, C., Kinemuchi, K., Ripepi, V., et al. 2008, ApJL, 674, L81

Laevens, B. P. M., Martin, N. F., Bernard, E. J., et al. 2015a, ApJ, 813, 44

Laevens, B. P. M., Martin, N. F., Ibata, R. A., et al. 2015b, ApJL, 802, L18

Layden, A. C. 1998 , AJ, 115, 193

Layden, A. C., \& Sarajedini, A. 2000, AJ, 119, 1760

Lee, Y.-W., Demarque, P., \& Zinn, R. 1990, ApJ, 350, 155

Lee, Y.-W., Demarque, P., \& Zinn, R. 1994, ApJ, 423, 248

Lim, D., Lee, Y.-W., Pasquato, M., Han, S.-I., \& Roh, D.-G. 2016, ApJ, 832,99

Mancone, C., \& Sarajedini, A. 2008, AJ, 136, 1913

Martin, N. F., de Jong, J. T. A., \& Rix, H.-W. 2008, ApJ, 684, 1075

Martin, N. F., Ibata, R. A., Chapman, S. C., Irwin, M., \& Lewis, G. F. 2007, MNRAS, 380, 281 
Martin, N. F., Nidever, D. L., Besla, G., et al. 2015, ApJL, 804, L5

Mateo, M., Hurley-Keller, D., \& Nemec, J. 1998, AJ, 115, 1856

McConnachie, A. W. 2012, AJ, 144, 4

McGaugh, S. S. 2016, ApJL, 832, L8

McNamara, D. H. 2011, AJ, 142, 110

Medina, G. E., Muñoz, R. R., Vivas, A. K., et al. 2017, ApJL, 845, L10

Monelli, M., Pulone, L., Corsi, C. E., et al. 2003, AJ, 126, 218

Moore, B., Ghigna, S., Governato, F., et al. 1999, ApJL, 524, L19

Moretti, M. I., Dall'Ora, M., Ripepi, V., et al. 2009, ApJL, 699, L125

Muñoz, R. R., Carlin, J. L., Frinchaboy, P. M., et al. 2006, ApJL, 650, L51

Muñoz, R. R., Geha, M., \& Willman, B. 2010, AJ, 140, 138

Musella, I., Ripepi, V., Clementini, G., et al. 2009, ApJL, 695, L83

Musella, I., Ripepi, V., Marconi, M., et al. 2012, ApJ, 756, 121

Nemec, J. M. 1985, AJ, 90, 204

Norris, J. E., Wyse, R. F. G., Gilmore, G., et al. 2010, ApJ, 723, 1632

Okamoto, S., Arimoto, N., Yamada, Y., \& Onodera, M. 2012, ApJ, 744, 96

Piersimoni, A. M., Bono, G., \& Ripepi, V. 2002, AJ, 124, 1528

Pietrukowicz, P., Kozłowski, S., Skowron, J., et al. 2015, ApJ, 811, 113

Pritzl, B. J., Armandroff, T. E., Jacoby, G. H., \& Da Costa, G. S. 2002, AJ, 124, 1464

Reimers, D. 1977, A\&A, 57, 395

Sakamoto, T., \& Hasegawa, T. 2006, ApJL, 653, L29

Sand, D. J., Strader, J., Willman, B., et al. 2012, ApJ, 756, 79

Sandage, A. 1993, AJ, 106, 703

Sandage, A. 2006, AJ, 131, 1750

Sarajedini, A., Barker, M. K., Geisler, D., Harding, P., \& Schommer, R. 2006 AJ, 132, 1361

Schlegel, D. J., Finkbeiner, D. P., \& Davis, M. 1998, ApJ, 500, 525

Sesar, B., Banholzer, S. R., Cohen, J. G., et al. 2014, ApJ, 793, 135

Shanks, T., Metcalfe, N., Chehade, B., et al. 2015, MNRAS, 451, 4238

Siegel, M. H. 2006, ApJL, 649, L83

Simon, J. D., \& Geha, M. 2007, ApJ, 670, 313

Simon, J. D., Geha, M., Minor, Q. E., et al. 2011, ApJ, 733, 46

Smith, H. A. 2004, RR Lyrae Stars, Cambridge Astrophysics (Cambridge: Cambridge Univ. Press)

Smith, H. A., Catelan, M., \& Kuehn, C. 2011, in RR Lyrae Stars, Metal-Poor Stars, and the Galaxy, Vol. 5 ed. A. McWilliam (Pasadena, CA: The Observatories of the Carnegie Institution of Washington), 17
Soszyński, I., Udalski, A., Szymański, M. K., et al. 2008, AcA, 58, 293

Stetson, P. B. 1987, PASP, 99, 191

Stetson, P. B. 1994, PASP, 106, 250

Stetson, P. B., Fiorentino, G., Bono, G., et al. 2014, PASP, 126, 616

Sturch, C. 1966, ApJ, 143, 774

Torrealba, G., Koposov, S. E., Belokurov, V., \& Irwin, M. 2016a, MNRAS, 459, 2370

Torrealba, G., Koposov, S. E., Belokurov, V., et al. 2016b, MNRAS, 463, 712 van Albada, T. S., \& Baker, N. 1971, ApJ, 169, 311

Vargas, L. C., Geha, M., Kirby, E. N., \& Simon, J. D. 2013, ApJ, 767, 134

Vivas, A. K., Olsen, K., Blum, R., et al. 2016, AJ, 151, 118

Walker, A. R. 1990, AJ, 100, 1532

Walker, A. R. 1998, AJ, 116, 220

Walsh, S. M., Jerjen, H., \& Willman, B. 2007, ApJL, 662, L83

Watkins, L. L., Evans, N. W., Belokurov, V., et al. 2009, MNRAS, 398, 1757

Willman, B., Blanton, M. R., West, A. A., et al. 2005a, AJ, 129, 2692

Willman, B., Dalcanton, J. J., Martinez-Delgado, D., et al. 2005b, ApJL, 626, L85

Willman, B., Governato, F., Dalcanton, J. J., Reed, D., \& Quinn, T. 2004, MNRAS, 353, 639

Willman, B., \& Strader, J. 2012, AJ, 144, 76

Yang, S.-C., \& Sarajedini, A. 2012, MNRAS, 419, 1362

Yang, S.-C., Sarajedini, A., Holtzman, J. A., \& Garnett, D. R. 2010, ApJ, 724,799

Yang, S.-C., Wagner-Kaiser, R., Sarajedini, A., Kim, S. C., \& Kyeong, J. 2014, ApJ, 784, 76

Yi, S. K., Kim, Y.-C., Demarque, P., et al. 2008, in IAU Symp. 252, The Art of Modeling Stars in the 21st Century, ed. L. Deng \& K.-L. Chan (Cambridge: Cambridge Univ. Press), 413

Yoon, S.-J., Joo, S.-J., Ree, C. H., et al. 2008, ApJ, 677, 1080

York, D. G., Adelman, J., Anderson, J. E., Jr., et al. 2000, AJ, 120, 1579

Zacharias, N., Finch, C., Girard, T., et al. 2010, AJ, 139, 2184

Zinn, R., Horowitz, B., Vivas, A. K., et al. 2014, ApJ, 781, 22

Zinn, R., \& West, M. J. 1984, ApJS, 55, 45

Zorotovic, M., Catelan, M., Smith, H. A., et al. 2010, AJ, 139, 357

Zucker, D. B., Belokurov, V., Evans, N. W., et al. 2006a, ApJL, 650, L41

Zucker, D. B., Belokurov, V., Evans, N. W., et al. 2006b, ApJL, 643, L103 